\title{
Optimal tests for random effects in linear mixed models
}

\author{
Yassine Ou Larbi*1 (D), Rachid El Halimi ${ }^{1}$ (D), Abdelhadi Akharif ${ }^{1}$ (D), Amal \\ Mellouk ${ }^{2}$ iD \\ ${ }^{1}$ Laboratoire de Mathématiques et Applications, Faculté des Sciences et Techniques, Abdelmalek Essaâdi \\ University, Tangier, Morocco \\ ${ }^{2}$ Centre Régional des Métiers de l'Education et de la Formation, Tangier, Morocco
}

\begin{abstract}
In the past decade, mixed-effects modeling has received a great deal of attention in the applied and theoretical statistical literature. They are very flexible tools in analyzing repeated measures, panel data, cross-sectional data, and hierarchical data. However, the complex nature of these models has motivated researchers to study different aspects of this problem. One of which is to test the significance of random effects used to model unobserved heterogeneity in the population. The method of likelihood ratio test based on the normality assumption of the error term and random effects has been proposed. However, this assumption does not necessarily hold in practice. In this paper, we propose an optimal test based on the so-called uniform local asymptotic normality to detect the possible presence of random effects in linear mixed models. We show that the proposed test statistic is, consistent, locally asymptotically optimal even for a model that does not require the traditional assumption of normality and is comparable to the classical L.ratiotest when the standard assumptions are met. Finally, simulation studies and real data analysis are also conducted to empirically examine the performance of this procedure.
\end{abstract}

Mathematics Subject Classification (2020). 62J05, 62F03, 62F05, 62 G10

Keywords. Linear mixed model, local asymptotic normality, pseudo-Gaussian test, rank tests

\section{Introduction}

The linear mixed-effects (LME) modeling (e.g.[16]) has been generating increasing interest in current statistical literature in last years $[6,25]$. These models are widely used to describe heterogeneity in a population and suitable to analyze repeated measures and hierarchical data in a wide variety of fields, such as health sciences, biology, economics, and pharmacokinetics. This because, both the intra and inter-subject variability in data with possible correlation structures can be modeled with appropriate random effects in addition to the error terms (e.g.[27]). They offer a suitable balance between over-parameterized

\footnotetext{
*Corresponding Author.

Email addresses: yassineoularbi2@gmail.com (Y. Ou Larbi), relhalimi@gmail.com (R. El Halimi), aakharif@gmail.com (A. Akharif), prmelloukamal@gmail.com (A. Mellouk)

Received: 25.07.2020; Accepted: 01.05.2021
} 
models separately fitted to each individual, and global models that do not take into account inter-individual parameter variability. A crucial issue when adjusting such a model to data consists of identifying fixed and/or random effects. Testing the nullity of the variances of random effects can be useful for checking unobserved heterogeneity of the population, which means determining whether there are significant individual-specific deviations from the population mean. In this context, one way to do so is through a standard likelihood ratio tests (LRT), as suggested by Morrell [20]. However, this test is based on the assumption that the random effects and the error terms follow a multivariate normal distribution, which is not always the case in reality. More recently, this approach has been considered by several authors in conjunction with empirical Bayesian and permutation test (e.g.[24]) while Drikvandi and Noorian [7] have considered the permutation test for a more broad class of linear mixed models with correlated errors. The results were shown that both tests to perform well, albeit the permutation test with the likelihood ratio statistic tends to provide a relatively higher power when testing multiple random effects. Our study is, to some extent, complementary to this paper. As an alternative to LR-test, Bayesian and permutation test, particularly concerning detecting the randomness in the coefficients of individual effects in longitudinal and clustered data, we present a parametric and non-parametric test locally and asymptotically optimal. Practical examples of a model building using Uniform Local Asymptotic Normality (ULAN) optimal test models can be found in $[1,4,10,19]$ among many others.

In this paper, the problem of testing random-slope model are studied, including the situation when the assumption of normality of random effects and error components is not met, we consider the specific model of the following form:

$$
Y_{i j}=\beta_{0}+\left(\beta_{1}+\eta_{i}\right) X_{i j}+\varepsilon_{i j}, \quad i=1, \ldots, n, \quad j=1, \ldots, m,
$$

where

- $Y_{i j}$ is the observed response for $j t h$ observation of individual $i$,

- $X_{i j}$ is a non-stochastic exogenous regressor,

- $\beta_{0}$ and $\beta_{1}$ are, respectively, the fixed effects for the intercept and the slope,

- $\varepsilon_{i j}$ is an $i . i . d$ error terms of sequence unobserved with probability density $f: \varepsilon \mapsto f(\varepsilon):=(1 / \sigma) f_{1}(\varepsilon / \sigma)$,

- $\eta_{i}$ is an i.i.d unobserved sequence of random effects with zero mean, $\sigma_{\eta}^{2}$ variance and density $h: \eta \mapsto h(\eta):=\left(1 / \sigma_{\eta}\right) h_{1}\left(\eta / \sigma_{\eta}\right)$,

- $\eta_{i}$ and $\varepsilon_{i j}$ are independent for all $i$ and $j$.

Under the null hypothesis the model (1.1) is reduced to

$$
Y_{i j}=\beta_{0}+\beta_{1} X_{i j}+\varepsilon_{i j}, \quad i=1, \ldots, n, \quad j=1, \ldots, m .
$$

It is therefore, important to be able to conduct a preliminary test of the null hypothesis $\mathcal{H}_{0}: \sigma_{\eta}^{2}=0$, with unspecified $\beta_{0}, \beta_{1}$, and $f$ versus the alternative $\mathcal{H}_{1}: \sigma_{\eta}^{2}>0$ (still, with unspecified $\beta_{0}, \beta_{1}, f$, and $h$ ).

The Likelihood Ratio Test (LRT) statistic is an asymptotic test statistic which has an $\chi^{2}$ distribution with degrees of freedom given by the difference in the number of parameters between the alternative and null hypotheses (see [11] and [20]), the test statistics (using REML log-likelihood) is

$$
L R T=2\left(L_{1}-L_{0}\right)
$$

where $L_{1}$ is the log-likelihood of the alternative hypothesis and $L_{0}$ is the log-likelihood of the null hypothesis.

Note that the LRT test is built with $f_{1}$ Gaussian. In the same sense, we try to derive parametric test using the ULAN. Of particular interest is the Gaussian test (where its square coincides with the LRT), with the proper standardization, we show that this test is valid on the class of all densities $f_{1}$ with finished fourth-order moments. 
The procedures described above require specified- $f_{1}$. These procedures are, therefore highly parametric. However, this parameter is generally unknown, and should, therefore, be considered a nuisance parameter. In order to eliminate this nuisance, we use a principle of invariance, and it is in this context that tools such as rank tests appear (van der Waerden, Wilcoxon and student).

The paper is organized as follows. In section 2.1, we collect the key assumptions and the main definitions. In section 2.2, we establish the result of ULAN. It allows us to build locally and asymptotically optimal parametric tests (Section 3.1). In section 3.2 we propose the special case of the pseudo-Gaussian test (optimal under Gaussian densities). These optimal parametric procedures are, however, only an intermediate step in the construction process (Sections 4.1 and 4.2) of the most important optimal rank-based optimal tests. Particular cases (van der Warden and Wilcoxon) are considered in section 4.4. We apply our test procedure to the real famous dental growth dataset from [23], using the $\mathrm{R}$ package lme4 [3]. The technical proofs are given in the Appendix.

\section{Uniform local asymptotic normality}

\subsection{Notation and basic assumptions}

To investigate the asymptotic behavior of the test statistics proposed and described below, first of all, we introduce the following notations and assumptions used throughout this document.

Let $\mathrm{P}_{\beta_{0}, \beta_{1}, \sigma^{2}, \sigma_{\eta}^{2} ; f_{1}, h_{1}}^{(n)}$ denotes the probability distribution of the sequence of observed variables $\mathbf{Y}^{(n)}:=\left(\mathbf{Y}_{1}^{(n)}, \mathbf{Y}_{2}^{(n)}, \ldots, \mathbf{Y}_{m}^{(n)}\right)^{\prime}$, where $\mathbf{Y}_{i}^{(n)}:=\left(Y_{i 1}^{(n)}, Y_{i 2}^{(n)}, \ldots, Y_{i m}^{(n)}\right)^{\prime}$ satisfying the regression model defined by equation (1.1), described above. In this formula, $h_{1}$ and $f_{1}$ stand for the standardized densities of the individual random effects and the errors, respectively. Under the null hypothesis $\left(\sigma_{\eta}^{2}=0\right)$, this last distribution reduced to $\mathrm{P}_{\beta_{0}, \beta_{1}, \sigma^{2}, 0 ; f_{1}}^{(n)}$. For a median zero and median absolute deviation one, we can consider the family of standardized densities:

$$
\mathcal{F}_{0}:=\left\{f_{1}: \int_{-1}^{1} f_{1}(z) d z=0.5=\int_{-\infty}^{0} f_{1}(z) d z\right\},
$$

which has no effect on our testing procedure and does not require any moment conditions, see [10] for example of such standardized densities.

The derivation of locally and asymptotically optimal tests at a given $f_{1}$ of density will be based on the ULAN, with respect to $\left(\beta_{0}, \beta_{1}, \sigma^{2}, \sigma_{\eta}^{2}\right)^{\prime}$, at $\left(\beta_{0}, \beta_{1}, \sigma^{2}, 0\right)^{\prime}$ of the families of distributions

$$
\mathcal{P}_{f_{1}, h_{1}}^{(n)}:=\left\{\mathrm{P}_{\beta_{0}, \beta_{1}, \sigma^{2}, \sigma_{\eta}^{2} ; f_{1}, h_{1}}^{(n)}:\left(\beta_{0}, \beta_{1}\right)^{\prime} \in \mathbb{R}^{2}, \sigma^{2}>0 \text { and } \sigma_{\eta}^{2} \geq 0\right\} .
$$

An important precaution for ULAN property is that some regularity conditions must be imposed which go back to [13]. Summarizing this, we throughout assume that the following assumption holds:

Assumption (A) The density $f_{1}$ is such that

(A.1) $f_{1} \in \mathcal{F}_{0}$,

(A.2) $f_{1}(z)>0$ for all $z \in \mathbb{R}$,

(A.3) $f_{1}$ is $C^{2}$, with derivatives $\dot{f}_{1}$ and $\ddot{f}_{1}$; letting $\phi_{f_{1}}:=-\dot{f}_{1} / f_{1}$ and $\psi_{f_{1}}:=\ddot{f}_{1} / f_{1}$, assume that

$$
\begin{gathered}
\mathcal{I}_{\phi}\left(f_{1}\right):=\int_{\mathbb{R}} \phi_{f_{1}}^{2}(z) f_{1}(z) d z<\infty, \quad \mathcal{I}_{\psi}\left(f_{1}\right):=\int_{\mathbb{R}} \psi_{f_{1}}^{2}(z) f_{1}(z) d z<\infty, \\
\mathcal{J}_{\phi}\left(f_{1}\right):=\int_{\mathbb{R}} z^{2} \phi_{f_{1}}^{2}(z) f_{1}(z) d z<\infty, \quad \text { and } \mathcal{K}_{\phi \phi}\left(f_{1}\right):=\int_{\mathbb{R}} z \phi_{f_{1}}^{2}(z) f_{1}(z) d z<\infty .
\end{gathered}
$$


Note that (A3) automatically also entails

$$
\mathcal{I}_{\phi \psi}\left(f_{1}\right):=\int_{\mathbb{R}} \phi_{f_{1}}(z) \psi_{f_{1}}(z) f_{1}(z) d z<\infty \text { and } \mathcal{K}_{\phi \psi}\left(f_{1}\right):=\int z \phi_{f_{1}}(z) \psi_{f_{1}}(z) f_{1}(z) d z<\infty \text {. }
$$

Denote by $\mathcal{F}_{A}$ the class of all densities satisfying Assumption (A), note that for any $f_{1} \in \mathcal{F}_{A}$,

(i) $\int \phi_{f_{1}}(z) f_{1}(z) d z=\int z^{2} \phi_{f_{1}}(z) f_{1}(z) d z=\int \psi_{f_{1}}(z) f_{1}(z) d z=\int z \psi_{f_{1}}(z) f_{1}(z) d z=0$,

(ii) $\int z \phi_{f_{1}}(z) f_{1}(z) d z=1$ and $\int z^{2} \psi_{f_{1}}(z) f_{1}(z) d z=2$,

(iii) the mapping $\theta \mapsto \boldsymbol{\Gamma}_{f_{1}}(\theta)$ is continuous for all $\theta \in \mathbb{R}^{2} \times \mathbb{R}_{0}^{+} \times \mathbb{R}^{+}$.

The following assumption concerns the asymptotic behavior of regression coefficients, it is standard in the context of rank-based inference. Let

$$
\begin{gathered}
M_{k}^{(n)}:=\frac{1}{n m} \sum_{i=1}^{n} \sum_{j=1}^{m}\left(X_{i j}^{(n)}\right)^{k}, \quad k=1, \ldots, 4 \\
\bar{X}_{i \bullet}^{(n) 2}=\frac{1}{m} \sum_{j=1}^{m}\left(X_{i j}^{(n)}\right)^{2} \text { and } \overline{\bar{X}}_{\bullet \bullet}^{(n) 2}:=\frac{1}{n} \sum_{i=1}^{n}\left(\bar{X}_{i \bullet}^{(n) 2}\right)^{2}
\end{gathered}
$$

Assumption (B) The covariates $X_{i j}=X_{i j}^{(n)}, i=1, \ldots n$ and $j=1, \ldots, m$ are such that, (B.1) the classical Noether [21] condition here holds: namely,

$$
\lim _{n \rightarrow \infty}\left[\max _{1 \leq i \leq n}\left(X_{i j}^{(n)}-\bar{X}^{(n)}\right)^{2} / \sum_{i=1}^{n} \sum_{j=1}^{m}\left(X_{i j}^{(n)}-\bar{X}^{(n)}\right)^{2}\right]=0, \quad j=1, \ldots, m,
$$

(B.2) the sequence $M_{4}^{(n)}$ is bounded as $n \rightarrow \infty$,

(B.3) $\overline{\bar{X}}_{\bullet \bullet}^{(n) 2}$ and $M_{k}^{(n)}$ converge to $\mu_{2}^{\bar{X}_{\bullet \bullet}^{2}}$ and $\mu_{k}^{X}$, respectively, $k=1, \ldots, 4$; particularly, $M_{1}^{(n)}=\bar{X}^{(n)}$ converges to $\mu_{1}^{X}$.

Note that, asymptotic results hold under (B.1)-(B.2), as $n \rightarrow \infty$. But those requiring the convergence of local experiment to obtain ULAN property, only hold as $\mathrm{n}$ tends to infinity under (B.3).

Assumption (C) Hypothesis (C) concerns density (normalized) $h_{1}$ of random coefficient. Define $G_{\mathbf{z}, \mathbf{x}}(\eta, y)=\prod_{j=1}^{m} f_{1}\left(z_{j}-x_{j} y \eta\right), \ddot{G}_{\mathbf{z}, \mathbf{x}}(\eta, y):=\frac{\partial^{2} G_{\mathbf{z}, \mathbf{x}}(\eta, y)}{\partial y^{2}}$ for $y>0, \mathbf{x}=$ $\left(x_{1}, x_{2}, \ldots, x_{m}\right)^{\prime} \in \mathbb{R}^{m}, \mathbf{x}^{2}=\left(x_{1}^{2}, x_{2}^{2}, \ldots, x_{m}^{2}\right)^{\prime} \in \mathbb{R}^{m}$ and, $\mathbf{z}=\left(z_{1}, z_{2}, \ldots, z_{m}\right)^{\prime} \in \mathbb{R}^{m}$.

(C.1) $\int_{\mathbb{R}} \eta h_{1}(\eta) d \eta=0$ and $\int_{\mathbb{R}} \eta^{2} h_{1}(\eta) d \eta=1$,

(C.2) the Fisher information associated with $\sigma_{\eta}$ is

$$
\mathcal{I}_{\psi \phi}^{\mathbf{x}}\left(f_{1} ; y\right):= \begin{cases}\frac{1}{y^{2}} \int_{\mathbb{R}^{m}} \frac{\left[\int_{w=0}^{y} \int_{\mathbb{R}} \ddot{G}_{\mathbf{z}, \mathbf{x}}(\eta, w) h_{1}(\eta) d \eta d w\right]^{2}}{\int_{\mathbb{R}} \prod_{j=1}^{m} f_{1}\left(z_{j}-x y \eta\right) h_{1}(\eta) d \eta} d \mathbf{z} & \text { if } y>0 \\ m \overline{\mathbf{x}^{2}} \mathcal{I}_{\psi}\left(f_{1}\right)+m \mathcal{I}_{\phi}^{2}\left(f_{1}\right)\left(m(\overline{\mathbf{x}})^{2}-\overline{\mathbf{x}^{2}}\right) & \text { if } y=0 .\end{cases}
$$

Note that the function $y \mapsto \mathcal{I}_{\psi \phi}^{\mathbf{x}}\left(f_{1} ; y\right)$ is continuous from the right at $y=0$ for all $\mathbf{x}=\left(x_{1}, x_{2}, \ldots, x_{m}\right)^{\prime}$.

Assumption (C2) actually is an assumption involving the couple $\left(f_{1}, h_{1}\right)$ for all $f_{1} \in \mathcal{F}_{A}$, let

$$
\mathcal{F}_{C \mid f_{1}}:=\left\{h_{1} \mid h_{1} \text { satisfies (C.1) and }\left(f_{1}, h_{1}\right) \text { satisfy (C.2) }\right\} \text {. }
$$




\subsection{Uniform local asymptotic normality}

In the following, for a fixed density $f_{1} \in \mathcal{F}_{A}$, we establish the ULAN result with respect to intercept, regression coefficient, scale parameter $\sigma^{2}$, and the parameter of interest $\sigma_{\eta}^{2}=$ 0 , the reader is referred to [18].

We denote by $\mathcal{K}_{2}^{(n)}:=\left(M_{2}^{(n)}\right)^{-1 / 2}, \mathcal{K}_{4}^{(n)}:=\left(M_{4}^{(n)}\right)^{-1 / 2}$, and $\theta+n^{-1 / 2} \boldsymbol{\xi}^{(n)} \boldsymbol{\tau}^{(n)}$ sequences by small perturbations of the parameter $\theta:=\left(\beta_{0}, \beta_{1}, \sigma^{2}, 0\right)^{\prime}$ under alternative where,

$$
\boldsymbol{\xi}^{(n)}:=\left(\begin{array}{cccc}
1 & 0 & 0 & 0 \\
0 & \mathcal{K}_{2}^{(n)} & 0 & 0 \\
0 & 0 & 1 & 0 \\
0 & 0 & 0 & \mathcal{K}_{4}^{(n)}
\end{array}\right)
$$

and $\boldsymbol{\tau}^{(n)}:=\left(\tau_{1}^{(n)}, \tau_{2}^{(n)}, \tau_{3}^{(n)}, \tau_{4}^{(n)}\right)^{\prime} \in \mathbb{R}^{3} \times \mathbb{R}^{+}$is such that $\sup _{n}\left(\boldsymbol{\tau}^{(n)}\right)^{\prime} \boldsymbol{\tau}^{(n)}<\infty$.

Define the standardized residual $Z_{i j}$ by

$$
Z_{i j}=Z_{i j}\left(\beta_{0}, \beta_{1}, \sigma^{2}\right):=\frac{\left(Y_{i j}-\beta_{0}-\beta_{1} X_{i j}\right)}{\sigma}, \quad i=1, \ldots, n, \quad j=1, \ldots, m,
$$

and note that, under $\mathrm{P}_{\beta_{0}, \beta_{1}, \sigma^{2}, 0 ; f_{1}}^{(n)}, Z_{i j}$ coincides with $\varepsilon_{i j} / \sigma$. We then have the following proposition (see Appendix (A) for a proof).

Proposition 2.1 (ULAN). Let Assumptions (B.1), (B.2) and (C) hold. Fix $f_{1} \in \mathcal{F}_{A}$ and $h_{1} \in \mathcal{F}_{C \mid f_{1}}$. Then, the family $\mathcal{P}_{f_{1}, h_{1}}^{(n)}$ is ULAN (for $n \longrightarrow \infty$ with fixed $m$ ) at any $\theta:=\left(\beta_{0}, \beta_{1}, \sigma^{2}, 0\right)^{\prime}$ with central sequence

$$
\boldsymbol{\Delta}_{f_{1}}^{(n)}(\theta):=\left(\begin{array}{c}
\Delta_{f_{1} ; 1}^{(n)}(\theta) \\
\Delta_{f_{1} ; 2}^{(n)}(\theta) \\
\Delta_{f_{1} ; 3}^{(n)}(\theta) \\
\Delta_{f_{1} ; 4}^{(n)}(\theta)
\end{array}\right)=\frac{1}{\sigma \sqrt{n}}\left(\begin{array}{c}
\sum_{i=1}^{n} \sum_{j=1}^{m} \phi_{f_{1}}\left(Z_{i j}\right) \\
K_{2}^{(n)} \sum_{i=1}^{n} \sum_{j=1}^{m} \phi_{f_{1}}\left(Z_{i j}\right) X_{i j} \\
\frac{1}{2 \sigma} \sum_{i=1}^{n} \sum_{j=1}^{m}\left(Z_{i j} \phi_{f_{1}}\left(Z_{i j}\right)-1\right) \\
\frac{K_{4}^{(n)}}{2 \sigma} \sum_{i=1}^{n}\left\{\sum_{j=1}^{m} \psi_{f_{1}}\left(Z_{i j}\right) X_{i j}^{2}+\sum_{j=1}^{m} \sum_{\substack{l=1 \\
l \neq j}}^{m} \phi_{f_{1}}\left(Z_{i j}\right) \phi_{f_{1}}\left(Z_{i l}\right) X_{i j} X_{i l}\right\}
\end{array}\right)
$$

and information matrix

$$
\Gamma_{f_{1}}^{(n)}(\theta):=\frac{m}{\sigma^{2}}\left(\begin{array}{llll}
\Gamma_{f_{1} ; 11}^{(n)}(\theta) & \Gamma_{f_{1} ; 12}^{(n)}(\theta) & \Gamma_{f_{1} ; 13}^{(n)}(\theta) & \Gamma_{f_{1} ; 14}^{(n)}(\theta) \\
\Gamma_{f_{1} ; 12}^{(n)}(\theta) & \Gamma_{f_{1} ; 22}^{(n)}(\theta) & \Gamma_{f_{1} ; 23}^{(n)}(\theta) & \Gamma_{f_{1} ; 24}^{(n)}(\theta) \\
\Gamma_{f_{1} ; 13}^{(n)}(\theta) & \Gamma_{f_{1} ; 23}^{(n)}(\theta) & \Gamma_{f_{1} ; 33}^{(n)}(\theta) & \Gamma_{f_{1} ; 34}^{(n)}(\theta) \\
\Gamma_{f_{1} ; 14}^{(n)}(\theta) & \Gamma_{f_{1} ; 24}^{(n)}(\theta) & \Gamma_{f_{1} ; 34}^{(n)}(\theta) & \Gamma_{f_{1} ; 44}^{(n)}(\theta)
\end{array}\right)
$$

with

$$
\begin{aligned}
\Gamma_{f_{1} ; 11}^{(n)}(\theta) & :=\mathcal{I}_{\phi}\left(f_{1}\right), \quad \Gamma_{f_{1} ; 12}^{(n)}(\theta):=\frac{M_{1}^{(n)}}{\left(M_{2}^{(n)}\right)^{1 / 2}} \mathcal{I}_{\phi}\left(f_{1}\right), \quad \Gamma_{f_{1} ; 13}^{(n)}(\theta):=\frac{1}{2 \sigma} \mathcal{K}_{\phi \phi}\left(f_{1}\right), \\
\Gamma_{f_{1} ; 14}^{(n)}(\theta) & :=\frac{M_{2}^{(n)}}{2 \sigma\left(M_{4}^{(n)}\right)^{1 / 2}} \mathcal{I}_{\phi \psi}\left(f_{1}\right), \quad \Gamma_{f_{1} ; 22}^{(n)}(\theta):=\mathcal{I}_{\phi}\left(f_{1}\right), \quad \Gamma_{f_{1} ; 23}^{(n)}(\theta):=\frac{M_{1}^{(n)}}{2 \sigma\left(M_{2}^{(n)}\right)^{1 / 2}} \mathcal{K}_{\phi \phi}\left(f_{1}\right), \\
\Gamma_{f_{1} ; 24}^{(n)}(\theta) & :=\frac{M_{3}^{(n)}}{2 \sigma\left(M_{2}^{(n)} M_{4}^{(n)}\right)^{1 / 2}} \mathcal{I}_{\phi \psi}\left(f_{1}\right), \quad \Gamma_{f_{1} ; 34}^{(n)}(\theta):=\frac{M_{2}^{(n)}}{4 \sigma^{2}\left(M_{4}^{(n)}\right)^{1 / 2}} \mathcal{K}_{\phi \psi}\left(f_{1}\right), \\
\Gamma_{f_{1} ; 33}^{(n)}(\theta) & :=\frac{1}{4 \sigma^{2}}\left(\mathcal{J}_{\phi}\left(f_{1}\right)-1\right), \quad \text { and }
\end{aligned}
$$


$\Gamma_{f_{1} ; 44}^{(n)}(\theta):=\frac{1}{4 \sigma^{2}\left(M_{4}^{(n)}\right)}\left(\mathcal{I}_{\psi}\left(f_{1}\right) M_{4}^{(n)}+2 \mathcal{I}_{\phi}^{2}\left(f_{1}\right)\left(m \overline{\bar{X}}_{\bullet \bullet}^{(n) 2}-M_{4}^{(n)}\right)\right)$.

More specifically, for any sequence $\theta^{(n)}:=\left(\beta_{0}^{(n)}, \beta_{1}^{(n)},\left(\sigma^{(n)}\right)^{2}, 0\right)^{\prime}$, such that $n^{1 / 2}\left(\beta_{0}^{(n)}\right.$ $\left.\beta_{0}\right), n^{1 / 2}\left(\mathcal{K}_{2}^{(n)}\right)^{-1}\left(\beta_{1}^{(n)}-\beta_{1}\right)$ and $n^{1 / 2}\left(\left(\sigma^{(n)}\right)^{2}-\sigma^{2}\right)$ are $O(1)$. For any bounded sequence $\boldsymbol{\tau}^{(n)} \in \mathbb{R}^{3} \times \mathbb{R}^{+}$under $\mathrm{P}_{\theta^{(n)} ; f_{1}}^{(n)}$ (as $n \longrightarrow \infty$ with fixed $m$ ), we have

$$
\begin{aligned}
& \Lambda_{\theta^{(n)}+n^{-1 / 2} \boldsymbol{\xi}^{(n)} \boldsymbol{\tau}^{(n) / \theta(n)} ; f_{1}, h_{1}}^{(n)}:=\log \left(\frac{d \mathrm{P}_{\theta^{(n)}+n^{-1 / 2} \boldsymbol{\xi}^{(n)} \boldsymbol{\tau}^{(n)} ; f_{1}, h_{1}}^{(n)}}{d \mathrm{P}_{\theta^{(n)} ; f_{1}}^{(n)}}\right) \\
& =\boldsymbol{\tau}^{(n)^{\prime}} \boldsymbol{\Delta}_{f_{1}}^{(n)}\left(\theta^{(n)}\right)-\frac{1}{2} \tau^{(n)^{\prime}} \boldsymbol{\Gamma}_{f_{1}}^{(n)}(\theta) \boldsymbol{\tau}^{(n)}+o_{\mathrm{P}}(1)
\end{aligned}
$$

and

$$
\left(\boldsymbol{\Gamma}_{f_{1}}^{(n)}(\theta)\right)^{-1 / 2} \boldsymbol{\Delta}_{f_{1}}^{(n)}\left(\theta^{(n)}\right) \stackrel{\mathcal{L}}{\longrightarrow} \mathcal{N}(0, \mathbf{I})
$$

Let Assumption (B.3), $\boldsymbol{\Gamma}_{f_{1}}^{(n)}(\theta)$ converges to

$$
\Gamma_{f_{1}}(\theta):=\frac{m}{\sigma^{2}}\left(\begin{array}{cccc}
\mathcal{I}_{\phi}\left(f_{1}\right) & \frac{\mu_{1}^{X}}{\left(\mu_{2}^{X}\right)^{1 / 2}} \mathcal{I}_{\phi}\left(f_{1}\right) & \frac{1}{2 \sigma} \mathcal{K}_{\phi \phi}\left(f_{1}\right) & \frac{\mu_{2}^{X}}{2 \sigma\left(\mu_{4}^{X}\right)^{1 / 2}} \mathcal{I}_{\phi \psi}\left(f_{1}\right) \\
\frac{\mu_{1}^{X}}{\left(\mu_{2}^{X}\right)^{1 / 2}} \mathcal{I}_{\phi}\left(f_{1}\right) & \mathcal{I}_{\phi}\left(f_{1}\right) & \frac{\mu_{1}^{X}}{2 \sigma\left(\mu_{2}^{X}\right)^{1 / 2}} \mathcal{K}_{\phi \phi}\left(f_{1}\right) & \frac{\mu_{3}^{X}}{2 \sigma\left(\mu_{2}^{X} \mu_{4}^{X}\right)^{1 / 2}} \mathcal{I}_{\phi \psi}\left(f_{1}\right) \\
\frac{1}{2 \sigma} \mathcal{K}_{\phi \phi}\left(f_{1}\right) & \frac{\mu_{1}^{X}}{2 \sigma\left(\mu_{2}^{X}\right)^{1 / 2}} \mathcal{K}_{\phi \phi}\left(f_{1}\right) & \frac{1}{4 \sigma^{2}}\left(\mathcal{J}_{\phi}\left(f_{1}\right)-1\right) & \frac{\mu_{2}^{X}}{4 \sigma^{2}\left(\mu_{4}^{X}\right)^{1 / 2}} \mathcal{K}_{\phi \psi}\left(f_{1}\right) \\
\frac{\mu_{2}^{X}}{2 \sigma\left(\mu_{4}^{X}\right)^{1 / 2}} \mathcal{I}_{\phi \psi}\left(f_{1}\right) & \frac{\mu_{3}^{X}}{2 \sigma\left(\mu_{2}^{X} \mu_{4}^{X}\right)^{1 / 2}} \mathcal{I}_{\phi \psi}\left(f_{1}\right) & \frac{\mu_{2}^{X}}{4 \sigma^{2}\left(\mu_{4}^{X}\right)^{1 / 2}} \mathcal{K}_{\phi \psi}\left(f_{1}\right) & \Gamma_{f_{1} ; 44}(\theta)
\end{array}\right)
$$

where

$$
\Gamma_{f_{1} ; 44}(\theta):=\frac{1}{4 \sigma^{2}\left(\mu_{4}^{X}\right)^{1 / 2}}\left\{\mu_{4}^{X}\left(\mathcal{I}_{\psi}\left(f_{1}\right)-2 \mathcal{I}_{\phi}^{2}\left(f_{1}\right)\right)+2 m \mathcal{I}_{\phi}^{2}\left(f_{1}\right) \mu_{2}^{\bar{X}_{\bullet}^{2}}\right\}
$$

Return to $\boldsymbol{\Delta}_{f_{1}}^{(n)}(\theta)$, via Le Cam's Third Lemma, under $\mathrm{P}_{\theta+n^{-1 / 2} \boldsymbol{\xi}^{(n)} \boldsymbol{\tau} ; f_{1}, h_{1}}^{(n)}$, as $n \rightarrow \infty$, we can proof that:

$$
\boldsymbol{\Delta}_{f_{1}}^{(n)}(\theta) \stackrel{\mathcal{L}}{\longrightarrow} \mathcal{N}\left(\boldsymbol{\Gamma}_{f_{1}}(\theta) \boldsymbol{\tau}, \boldsymbol{\Gamma}_{f_{1}}(\theta)\right) .
$$

We have also the asymptotic relative linearity of central sequences, namely,

$$
\boldsymbol{\Delta}_{f_{1}}^{(n)}\left(\theta+\boldsymbol{\xi}^{(n)} \boldsymbol{\tau}\right)-\boldsymbol{\Delta}_{f_{1}}^{(n)}(\theta)=-\boldsymbol{\Gamma}_{f_{1}}^{(n)}(\theta) \boldsymbol{\tau}+o_{\mathrm{P}}(1)
$$

for all $\boldsymbol{\tau}$ under $\mathrm{P}_{\theta ; f_{1}}^{(n)}$, as $n \rightarrow \infty$, which allows us to estimate the unknown parameter $\theta$ in the test statistic.

The non-diagonal form of $\boldsymbol{\Gamma}_{f_{1}}^{(n)}(\theta)$ implies that the nuisance parameters $\beta_{0}, \beta_{1}, \sigma^{2}$, in general, are not information-orthogonal to the parameter of interest $\sigma_{\eta}^{2}$. Point out that the density $h_{1}$ of the random coefficient does not appear in the central sequence (2.3) and the information matrix (2.4). Therefore, it does not influence the optimal test statistics.

\section{Optimal parametric and pseudo-Gaussian tests}

We are interested in testing the null hypothesis of absence of random slope $\left(\sigma_{\eta}^{2}=0\right)$ in (1.1). It can be formally written

$$
\mathcal{H}_{0}^{(n)}:=\bigcup_{g_{1} \in \mathcal{F}_{0}} \mathcal{H}_{0}^{(n)}\left(g_{1}\right):=\bigcup_{g_{1} \in \mathcal{F}_{0}} \bigcup_{\beta_{0} \in \mathbb{R}} \bigcup_{\beta_{1} \in \mathbb{R}} \bigcup_{\sigma^{2}>0}\left\{\mathrm{P}_{\beta_{0}, \beta_{1}, \sigma^{2}, 0 ; g_{1}}^{(n)}\right\},
$$


where the (standardized) noise density remains an unspecified semiparametric hypothesis. Parametric alternatives will be considered, of the form, for a fixed density $f_{1} \in \mathcal{F}_{A}$,

$$
\mathcal{H}_{1}^{(n)}\left(f_{1}\right):=\bigcup_{\beta_{0} \in \mathbb{R}} \bigcup_{\beta_{1} \in \mathbb{R}} \bigcup_{\sigma^{2}>0} \bigcup_{\sigma_{\eta}^{2}>0} \bigcup_{h_{1} \in \mathcal{F}_{C \mid f_{1}}}\left\{\mathrm{P}_{\beta_{0}, \beta_{1}, \sigma^{2}, \sigma_{\eta}^{2} ; f_{1}, h_{1}}^{(n)}\right\} .
$$

The parameters $\beta_{0}, \beta_{1}$, and $\sigma^{2}$ are nuisance parameters, while $\sigma_{\eta}^{2}$ is the parameter of interest. Let us first study the problem parametric tests $\mathcal{H}_{0}^{(n)}\left(f_{1}\right)$ against $\mathcal{H}_{1}^{(n)}\left(f_{1}\right)$.

\subsection{Optimal parametric tests}

We suppose that the innovation density $f_{1}$ is specified, the main consequence of the ULAN results imply that the local experiments

$$
\left\{\mathrm{P}_{\theta+n^{-1 / 2} \boldsymbol{\xi}^{(n)} \boldsymbol{\tau} ; f_{1}, h_{1}}^{(n)} \mid \boldsymbol{\tau} \in \mathbb{R}^{3} \times \mathbb{R}^{+}, h_{1} \in \mathcal{F}_{C \mid f_{1}}\right\}
$$

converges to the Gaussian shift experiment $\left(\boldsymbol{\Gamma}_{f_{1}}\right.$ given in 2.7$)$

$$
\left\{\mathcal{N}\left(\boldsymbol{\Gamma}_{f_{1}}(\theta) \boldsymbol{\tau}, \boldsymbol{\Gamma}_{f_{1}}(\theta)\right) \mid \boldsymbol{\tau} \in \mathbb{R}^{3} \times \mathbb{R}^{+}\right\} .
$$

ULAN and this convergence imply that locally optimal test must be based on the residual of $\Delta_{4}$ with respect to $\left(\Delta_{1}, \Delta_{2}, \Delta_{3}\right)^{\prime}$, computed at $\Delta_{f_{1} ; 4}^{(n)}(\theta)$ and $\left(\Delta_{f_{1} ; 1}^{(n)}(\theta), \Delta_{f_{1} ; 2}^{(n)}(\theta), \Delta_{f_{1} ; 3}^{(n)}(\theta)\right)^{\prime}$ (see, for instance [17]). That residual takes the form

$$
\begin{aligned}
\Delta_{f_{1} ; 4}^{*(n)}(\theta) & :=\Delta_{f_{1} ; 4}^{(n)}(\theta)-\left(\Gamma_{f_{1} ; 14}(\theta), \Gamma_{f_{1} ; 24}(\theta), \Gamma_{f_{1} ; 34}(\theta)\right) \\
& \times\left(\begin{array}{lll}
\Gamma_{f_{1} ; 11}(\theta) & \Gamma_{f_{1} ; 12}(\theta) & \Gamma_{f_{1} ; 13}(\theta) \\
\Gamma_{f_{1} ; 12}(\theta) & \Gamma_{f_{1} ; 22}(\theta) & \Gamma_{f_{1} ; 23}(\theta) \\
\Gamma_{f_{1} ; 13}(\theta) & \Gamma_{f_{1} ; 23}(\theta) & \Gamma_{f_{1} ; 33}(\theta)
\end{array}\right)^{-1}\left(\begin{array}{c}
\Delta_{f_{1} ; 1}^{(n)}(\theta) \\
\Delta_{f_{1} ; 2}^{(n)}(\theta) \\
\Delta_{f_{1} ; 3}^{(n)}(\theta)
\end{array}\right) \\
& =\Delta_{f_{1} ; 4}^{(n)}(\theta)-\Gamma_{f_{1} ; 1}^{*}(\theta) \Delta_{f_{1} ; 1}^{(n)}(\theta)-\Gamma_{f_{1} ; 2}^{*}(\theta) \Delta_{f_{1} ; 2}^{(n)}(\theta)-\Gamma_{f_{1} ; 3}^{*}(\theta) \Delta_{f_{1} ; 3}^{(n)}(\theta)
\end{aligned}
$$

$\Delta_{f_{1} ; 4}^{*(n)}(\theta)$ is asymptotically normal under $\mathrm{P}_{\theta ; f_{1}}^{(n)}$ with mean zero and variance

$$
\begin{aligned}
& \Gamma_{f_{1} ; 44}^{*}(\theta):=\Gamma_{f_{1} ; 44}(\theta)-\left(\Gamma_{f_{1} ; 14}(\theta), \Gamma_{f_{1} ; 24}(\theta), \Gamma_{f_{1} ; 34}(\theta)\right) \\
& \times\left(\begin{array}{lll}
\Gamma_{f_{1} ; 11}(\theta) & \Gamma_{f_{1} ; 12}(\theta) & \Gamma_{f_{1} ; 13}(\theta) \\
\Gamma_{f_{1} ; 12}(\theta) & \Gamma_{f_{1} ; 22}(\theta) & \Gamma_{f_{1} ; 23}(\theta) \\
\Gamma_{f_{1} ; 13}(\theta) & \Gamma_{f_{1} ; 23}(\theta) & \Gamma_{f_{1} ; 33}(\theta)
\end{array}\right)^{-1}\left(\begin{array}{l}
\Gamma_{f_{1} ; 14}(\theta) \\
\Gamma_{f_{1} ; 24}(\theta) \\
\Gamma_{f_{1} ; 34}(\theta)
\end{array}\right) \\
& =\Gamma_{f_{1} ; 44}(\theta)-\Gamma_{f_{1} ; 1}^{*}(\theta) \Gamma_{f_{1} ; 14}(\theta)-\Gamma_{f_{1} ; 2}^{*}(\theta) \Gamma_{f_{1} ; 24}(\theta)-\Gamma_{f_{1} ; 3}^{*}(\theta) \Gamma_{f_{1} ; 34}(\theta) .
\end{aligned}
$$

Therefore a test locally uniformly asymptotically most powerful for the sequence $\left\{\mathrm{P}_{\beta_{0}, \beta_{1}, \sigma^{2}, 0 ; f_{1}}^{(n)}\right\}$ of null hypotheses against the alternatives $\left\{\mathrm{P}_{\beta_{0}, \beta_{1}, \sigma^{2}, \sigma_{\eta}^{2} ; f_{1}, h_{1}}^{(n)} \mid \sigma_{\eta}^{2}>0, h_{1} \in\right.$ $\left.\mathcal{F}_{C \mid f_{1}}\right\}$ can be based on the test statistic $T_{f_{1}}^{*(n)}(\theta)$, where

$$
T_{f_{1}}^{*(n)}(\theta):=\left(\Gamma_{f_{1} ; 44}^{*(n)}(\theta)\right)^{-1 / 2} \Delta_{f_{1} ; 4}^{*(n)}(\theta) .
$$

Recall that $\theta$ remains unknown, so it should be replaced by an adequate estimator $\hat{\theta}^{(n)}$. Thus, let us show that

$$
T_{f_{1}}^{*(n)}(\theta)-T_{f_{1}}^{*(n)}\left(\hat{\theta}^{(n)}\right)=o_{\mathrm{P}}(1) \quad \text { under } \mathrm{P}_{\theta ; f_{1}}^{(n)} \text {, as } n \rightarrow \infty
$$

where, $\theta=\left(\beta_{0}, \beta_{1}, \sigma^{2}, 0\right)^{\prime}$ and $\hat{\theta}^{(n)}=\left(\hat{\beta}_{0}^{(n)}, \hat{\beta}_{1}^{(n)}, \hat{\sigma}^{2(n)}, 0\right)^{\prime}$ satisfies the following assumptions. 


\section{Assumption (D)}

(D.1) $n^{1 / 2}\left(\boldsymbol{\xi}^{(n)}\right)^{-1}\left(\hat{\theta}^{(n)}-\theta\right)=O_{\mathrm{P}}(1)$ under any $\mathrm{P}_{\theta ; f_{1}}^{(n)}$, as $n \rightarrow \infty$,

(D.2) $\hat{\theta}^{(n)}$ is locally asymptotically discrete, that is, let us denote by $\mathcal{B}(r)$ the ball with radius $r$ and center at the origin, the number of possible values of $\hat{\theta}^{(n)}$ in shrinking ellipsoids of the form $\theta+\boldsymbol{\xi}^{(n)} \mathcal{B}(r)$ is finite.

Assumption (D.1) is a constancy assumption of the optimal rate, always under the null hypothesis. As also the assumption (D.2), it is standard in this context; in fact, any satisfactory estimator (D.1) can be converted into a satisfactory estimator (D.1) - (D.2) by discretization its three components first on mesh grids $c n^{-1 / 2}, c n^{-1 / 2} \mathcal{K}_{2}^{(n)}$ and $c n^{-1 / 2}$, respectively ( $c>0$ arbitrary).

Given the hypothesis (D.1), in the definition of $T_{f_{1}}^{*(n)}$, the continuous mapping theorem, therefore, implies that replacing $\left(\Gamma_{f_{1} ; 44}^{*(n)}(\theta)\right)$ with $\left(\Gamma_{f_{1} ; 44}^{*(n)}\left(\hat{\theta}^{(n)}\right)\right)$ only has $o_{\mathrm{P}}(1)$ impact.

Proof. of (3.5). Recall that ULAN implies (2.8) for all $\boldsymbol{\tau}^{(n)}$, due to (D.1)-(D.2), that gives way, under $\mathrm{P}_{\theta ; f_{1}}^{(n)}$, as $n \rightarrow \infty$,

$$
\begin{aligned}
& \Delta_{f_{1} ; 4}^{*(n)}\left(\hat{\theta}^{(n)}\right)-\Delta_{f_{1} ; 4}^{*(n)}(\theta) \\
& =\Delta_{f_{1} ; 4}^{(n)}\left(\hat{\theta}^{(n)}\right)-\Delta_{f_{1} ; 4}^{(n)}(\theta) \\
& -\left(\Gamma_{14}, \Gamma_{24}, \Gamma_{34}\right)\left(\begin{array}{ccc}
\Gamma_{11} & \Gamma_{12} & \Gamma_{13} \\
\Gamma_{21} & \Gamma_{22} & \Gamma_{23} \\
\Gamma_{31} & \Gamma_{32} & \Gamma_{33}
\end{array}\right)^{-1}\left(\begin{array}{c}
\Delta_{f_{1} ; 1}^{(n)}\left(\hat{\theta}^{(n)}\right)-\Delta_{f_{1 ;}(1)}^{(n)}(\theta) \\
\Delta_{f_{1 ;}\left(\theta^{n}\right)}^{(n)}\left(\hat{\theta}^{(n)}\right)-\Delta_{f_{1 ; 2}(\theta)}^{(n)}(\theta) \\
\Delta_{f_{1} ; 3}^{(n)}\left(\hat{\theta}^{(n)}\right)-\Delta_{f_{1} ; 3}^{(n)}(\theta)
\end{array}\right) \\
& =-\left(\Gamma_{41}, \Gamma_{42}, \Gamma_{43}\right)\left(\boldsymbol{\xi}^{(n)}\right)^{-1}\left(\begin{array}{c}
\hat{\beta}_{0}^{(n)}-\beta_{0} \\
\hat{\beta}_{1}^{(n)}-\beta_{1} \\
\left(\hat{\sigma}^{(n)}\right)^{2}-\sigma^{2}
\end{array}\right) \\
& +\left(\Gamma_{41}, \Gamma_{42}, \Gamma_{43}\right)\left(\begin{array}{lll}
\Gamma_{11} & \Gamma_{12} & \Gamma_{13} \\
\Gamma_{21} & \Gamma_{22} & \Gamma_{23} \\
\Gamma_{31} & \Gamma_{32} & \Gamma_{33}
\end{array}\right)^{-1} \\
& \times\left(\begin{array}{ccc}
\Gamma_{11} & \Gamma_{12} & \Gamma_{13} \\
\Gamma_{21} & \Gamma_{22} & \Gamma_{23} \\
\Gamma_{31} & \Gamma_{32} & \Gamma_{33}
\end{array}\right)\left(\boldsymbol{\xi}^{(n)}\right)^{-1}\left(\begin{array}{c}
\hat{\beta}_{0}^{(n)}-\beta_{0} \\
\hat{\beta}_{1}^{(n)}-\beta_{1} \\
\left(\hat{\sigma}^{(n)}\right)^{2}-\sigma^{2}
\end{array}\right)+o_{\mathrm{P}}(1) \\
& =o_{\mathrm{P}}(1) .
\end{aligned}
$$

Consequently,

$$
T_{f_{1}}^{*(n)}\left(\hat{\theta}^{(n)}\right)-T_{f_{1}}^{*(n)}(\theta)=o_{\mathrm{P}}(1) \quad \text { under } \mathrm{P}_{\theta ; f_{1}}^{(n)} \text {, as } n \rightarrow \infty .
$$

More precisely, from the application of Le Cam's third Lemma, we have the following result.

Proposition 3.1. Let $\hat{\theta}^{(n)}$ satisfy Assumptions (D), let Assumptions (B) and (C) hold, and fix $f_{1} \in \mathcal{F}_{A}$. Then,

(i) for any $\theta=\left(\beta_{0}, \beta_{1}, \sigma^{2}, 0\right)^{\prime}, T_{f_{1}}^{*(n)}\left(\hat{\theta}^{(n)}\right)$ is asymptotically normal, with mean zero under $\mathrm{P}_{\theta ; f_{1}}^{(n)}$, mean $\left(\Gamma_{f_{1} ; 44}^{*}(\theta)\right)^{1 / 2} \tau_{4}$ under $\mathrm{P}_{\theta+n^{-1 / 2} \boldsymbol{\xi}^{(n)} \boldsymbol{\tau} ; f_{1}, h_{1}}^{(n)}$, and variance one under both, 
(ii) The sequence of tests rejecting the null hypothesis $\mathcal{H}_{0}^{(n)}\left(f_{1}\right)$ as soon as $T_{f_{1}}^{*(n)}\left(\hat{\theta}^{(n)}\right)$ exceeds the $(1-\alpha)$ standard normal quantile of the standard normal distribution, is locally asymptotically most powerful unbiased, at asymptotic level $\alpha$, for $\mathcal{H}_{0}^{(n)}\left(f_{1}\right)$ against alternatives of the form

$$
\bigcup_{\beta_{0}} \bigcup_{\beta_{1}} \bigcup_{\sigma^{2}} \bigcup_{\sigma_{\eta}^{2}>0} \bigcup_{h_{1} \in \mathcal{F}_{C \mid f_{1}}}\left\{\mathrm{P}_{\beta_{0}, \beta_{1}, \sigma^{2}, \sigma_{\eta}^{2} ; f_{1}, h_{1}}^{(n)}\right\}
$$

An important case is the Gaussian versions $\left(f_{1}=\phi_{1}\right)$. It is easily verified that $(2.3)$ and (2.7) becomes

$$
\left.\boldsymbol{\Delta}_{\phi_{1}}^{(n)}(\theta)=\frac{a}{\sigma \sqrt{n}}\left(\begin{array}{c}
\sum_{i=1}^{n} \sum_{j=1}^{m} Z_{i j} \\
K_{2}^{(n)} \sum_{i=1}^{n} \sum_{j=1}^{m} Z_{i j} X_{i j} \\
\frac{1}{2 \sigma} \sum_{i=1}^{n} \sum_{j=1}^{m}\left(Z_{i j}^{2}-\frac{1}{a}\right) \\
\frac{a K_{4}^{(n)}}{2 \sigma} \sum_{i=1}^{n}\left\{\sum_{j=1}^{m}\left(Z_{i j}^{2}-\frac{1}{a}\right) X_{i j}^{2}+\sum_{j=1}^{m} \sum_{\substack{l=1 \\
l \neq j}}^{m} Z_{i j} Z_{i l} X_{i j} X_{i l}\right.
\end{array}\right\}\right),
$$

and

$$
\boldsymbol{\Gamma}_{\phi_{1}}(\theta)=\frac{m}{\sigma^{2}}\left(\begin{array}{cccc}
a & \frac{a \mu_{1}^{X}}{\left(\mu_{2}^{X}\right)^{1 / 2}} & 0 & 0 \\
\frac{a \mu_{1}^{X}}{\left(\mu_{2}^{X}\right)^{1 / 2}} & a & 0 & 0 \\
0 & 0 & \frac{1}{2 \sigma^{2}} & \frac{a \mu_{2}^{X}}{2 \sigma^{2}\left(\mu_{4}^{X}\right)^{1 / 2}} \\
0 & 0 & \frac{a \mu_{2}^{X}}{2 \sigma^{2}\left(\mu_{4}^{X}\right)^{1 / 2}} & \frac{m a^{2} \mu_{2}^{\bar{X}_{\bullet}}}{2 \sigma^{2}\left(\mu_{4}^{X}\right)^{1 / 2}}
\end{array}\right)
$$

\subsection{Pseudo-Gaussian test}

The Gaussian versions of (3.2) and (3.3), obtained from (3.6) and (3.7), are as follows

$$
\begin{gathered}
\Delta_{\phi_{1} ; 4}^{*(n)}(\theta)=\frac{a^{2} \mathcal{K}_{4}^{(n)}}{2 \sigma^{2} \sqrt{n}} \sum_{i=1}^{n}\left\{\sum_{j=1}^{m} Z_{i j}^{2}\left(X_{i j}^{2}-M_{2}^{(n)}\right)+\sum_{j=1}^{m} \sum_{\substack{l=1 \\
l \neq j}}^{m} Z_{i j} Z_{i l} X_{i j} X_{i l}\right\} \\
\Gamma_{\phi_{1} ; 44}^{*}(\theta)=\frac{a^{2}}{2 \sigma^{4} \mu_{4}^{X}}\left(m\left(m \mu_{2}^{X_{\bullet} \cdot \bullet}-\left(\mu_{2}^{X}\right)^{2}\right)\right) .
\end{gathered}
$$

The Gaussian central sequence $\Delta_{\phi_{1} ; 4}^{*(n)}(\theta)$ in (3.8) allows having optimal asymptotic tests under $g_{1}=\phi_{1}$. Define

$$
m_{1}^{(n)}:=\frac{1}{n m} \sum_{i=1}^{n} \sum_{j=1}^{m} Z_{i j}, \quad \mu_{1}\left(g_{1}\right):=\int z g_{1}(z) d z,
$$

and $\mu_{k}\left(g_{1}\right):=\int\left(z-\mu_{1}\left(g_{1}\right)\right)^{k} g_{1}(z) d z \quad$ for $\quad k=2,3,4$.

Let us show that the Gaussian optimal test is valid under densities $g_{1}$ in a broad class of densities, which is of course highly desirable. This is indeed possible, and that a small 
modification in (3.8) leads to a pseudo-Gaussian test, which remains valid when the actual density $g_{1} \in \mathcal{F}_{A}^{2}$, where $\mathcal{F}_{A}^{2}$ the class of all densities $g_{1} \in \mathcal{F}_{A}$ such that $\mu_{4}\left(g_{1}\right)<\infty$. Define $\Delta_{\phi_{1} ; 4}^{\bullet(n)}(\theta)=\frac{a^{2} \mathcal{K}_{4}^{(n)}}{2 \sigma^{2} \sqrt{n}} \sum_{i=1}^{n}\left\{\sum_{j=1}^{m} Z_{i j}^{2}\left(X_{i j}^{2}-M_{2}^{(n)}\right)+\sum_{j=1}^{m} \sum_{\substack{l=1 \\ l \neq j}}^{m}\left(Z_{i j}-m_{1}^{(n)}\right)\left(Z_{i l}-m_{1}^{(n)}\right) X_{i j} X_{i l}\right\}$.

Decomposing $\left(Z_{i j}-m_{1}^{(n)}\right)$ into $\left(Z_{i j}-\mu_{1}\left(g_{1}\right)\right)+\left(\mu_{1}\left(g_{1}\right)-m_{1}^{(n)}\right)$, it easily follows from this consistency that

$$
\Delta_{\phi_{1} ; 4}^{\bullet(n)}(\theta)=\frac{a^{2} \mathcal{K}_{4}^{(n)}}{2 \sigma^{2} \sqrt{n}} \sum_{i=1}^{n}\left\{\sum_{j=1}^{m} Z_{i j}^{2}\left(X_{i j}^{2}-M_{2}^{(n)}\right)+\sum_{j=1}^{m} \sum_{\substack{l=1 \\ l \neq j}}^{m}\left(Z_{i j}-\mu_{1}\left(g_{1}\right)\right)\left(Z_{i l}-\mu_{1}\left(g_{1}\right)\right) X_{i j} X_{i l}\right\}+o_{\mathrm{P}}(1) .
$$

Under $\mathrm{P}_{\theta ; g_{1}}^{(n)}$ (with $\left.g_{1} \in \mathcal{F}_{A}^{2}\right), \Delta_{\phi_{1} ; 4}^{\bullet(n)}(\theta)$ is asymptotically normal with mean zero and variance

$$
\Gamma_{\phi_{1}, g_{1} ; 44}^{\bullet}(\theta)=\frac{a^{4}}{4 \sigma^{4} \mu_{4}^{X}}\left(m\left(\mu_{4}^{X}-\left(\mu_{2}^{X}\right)^{2}\right)\left(\mu_{4}\left(g_{1}\right)-\left(\mu_{2}\left(g_{1}\right)\right)^{2}\right)+2 m\left(\mu_{2}\left(g_{1}\right)\right)^{2}\left(m \mu_{2}^{\overline{X_{\bullet}} \cdot}-\mu_{4}^{X}\right)\right) .
$$

Consequently under $\mathrm{P}_{\theta ; g_{1}}^{(n)}$, as $n \rightarrow \infty$, for any $g_{1} \in \mathcal{F}_{A}^{2}$,

$$
T_{\phi_{1}}^{\bullet(n)}(\theta):=\left(\Gamma_{\phi_{1}, g_{1} ; 44}^{\bullet}(\theta)\right)^{-1 / 2} \Delta_{\phi_{1} ; 4}^{\bullet(n)}(\theta)
$$

is asymptotically standard normal.

In practice, the pseudo-Gaussian test will be based on the statistics $T_{\phi_{1}}^{\bullet(n)}(\theta)$, when $\mu_{4}\left(g_{1}\right), \mu_{2}\left(g_{1}\right)$, and $\mu_{1}\left(g_{1}\right)$ replaced by $\frac{1}{n m} \sum_{i=1}^{n} \sum_{j=1}^{m}\left(Z_{i j}-m_{1}^{(n)}\right)^{4}, \frac{1}{n m} \sum_{i=1}^{n} \sum_{j=1}^{m}\left(Z_{i j}-m_{1}^{(n)}\right)^{2}$, and $\frac{1}{n m} \sum_{i=1}^{n} \sum_{j=1}^{m}\left(Z_{i j}-m_{1}^{(n)}\right)$, respectively.

The parameter $\theta$ remains unspecified under the null hypothesis, so it should be replaced by estimator $\hat{\theta}$. It means that under $\mathrm{P}_{\theta ; q_{1}}^{(n)}$, for any $g_{1} \in \mathcal{F}_{A}^{2}$,

$T_{\phi_{1}}^{\bullet(n)}\left(\hat{\theta}^{(n)}\right)-T_{\phi_{1}}^{\bullet(n)}(\theta)$ is $o_{\mathrm{P}}(1)$. For that, we have to show $\Delta_{\phi_{1} ; 4}^{*(n)}\left(\hat{\theta}^{(n)}\right)-\Delta_{\phi_{1} ; 4}^{*(n)}(\theta)$ is $o_{\mathrm{P}}(1)$.

Using the linearity of $\boldsymbol{\Delta}_{\phi_{1}}^{(n)}(\theta)$ under $\mathrm{P}_{\theta ; g_{1}}^{(n)}$, for any $\boldsymbol{\tau}=\left(\tau_{1}, \tau_{2}, \tau_{3}, 0\right)^{\prime}, g \in \mathcal{F}_{A}^{2}$,

$$
\boldsymbol{\Delta}_{\phi_{1}}^{(n)}\left(\theta+\boldsymbol{\xi}^{(n)} \boldsymbol{\tau}\right)-\boldsymbol{\Delta}_{\phi_{1}}^{(n)}(\theta)=-\boldsymbol{\Gamma}_{\phi_{1} ; g_{1}}^{(n)}(\theta) \boldsymbol{\tau}+o_{\mathrm{P}}(1) .
$$

Following the same steps as in proof of (3.5), we can easily find the equivalence between $\Delta_{\phi_{1} ; 4}^{*(n)}\left(\hat{\theta}^{(n)}\right)$ and $\Delta_{\phi_{1} ; 4}^{*(n)}(\theta)$, therefore between $T_{\phi_{1}}^{\bullet(n)}\left(\hat{\theta}^{(n)}\right)$ and $T_{\phi_{1}}^{\bullet(n)}(\theta)$. In summary, we have the following result.

Proposition 3.2. Let $\hat{\theta}^{(n)}$ satisfy Assumptions (D), let Assumptions (B) and (C) hold, for any $g_{1} \in \mathcal{F}_{A}^{2}$. Then,

(i) for any $\theta=\left(\beta_{0}, \beta_{1}, \sigma^{2}, 0\right)^{\prime}, T_{\phi_{1}}^{\bullet(n)}\left(\hat{\theta}^{(n)}\right)$ is asymptotically normal, with mean zero under $\mathrm{P}_{\theta ; g_{1}}^{(n)}$, mean $\left(\Gamma_{\phi_{1} ; 44}^{*}(\theta)\right)\left(\Gamma_{\phi_{1}, g_{1} ; 44}^{\bullet}(\theta)\right)^{-1 / 2} \tau_{4}$ under $\mathrm{P}_{\theta+n^{-1 / 2} \boldsymbol{\xi}^{(n)} \boldsymbol{\tau} ; g_{1}, h_{1}}^{(n)}$, and variance one under both,

(ii) The sequence of tests rejecting the null hypothesis $\mathcal{H}_{A}^{(n) 2}:=\bigcup_{g_{1} \in \mathcal{F}_{A}^{2}} \mathcal{H}_{0}^{(n)}\left(g_{1}\right)$ as soon as $T_{\phi_{1}}^{\bullet(n)}\left(\hat{\theta}^{(n)}\right)$ exceeds the $(1-\alpha)$ standard normal quantile of the standard normal distribution, is locally asymptotically most powerful unbiased, at asymptotic level $\alpha$, for $\mathcal{H}_{A}^{(n) 2}$ against alternatives of the form

$$
\bigcup_{\beta_{0}} \bigcup_{\beta_{1}} \bigcup_{\sigma^{2}} \bigcup_{\sigma_{\eta}^{2}>0} \bigcup_{h_{1} \in \mathcal{F}_{C \mid \phi_{1}}}\left\{\mathrm{P}_{\beta_{0}, \beta_{1}, \sigma^{2}, \sigma_{\eta}^{2} ; \phi_{1}, h_{1}}^{(n)}\right\} .
$$




\section{Optimal rank tests}

Regarding parametric tests obtained in Section 3.1, their drawback is that the validity of these tests is limited to $f_{1}$ must be specified, and for the pseudo-Gaussian test in Section 3.2 , it still needs finite order moments four.

Its performances are likely based on the actual underlying density. In practice, a correct specification of the actual density $g_{1}$ is totally unrealistic, the problem must be considered a semi-parametric standpoint, where $g_{1}$ plays the role of a nuisance, however, it is more delicate than in the parametric case (see [9]).

\subsection{From parametric to semiparametric experiments}

The central sequences $\boldsymbol{\Delta}_{f_{1}}^{(n)}(\theta)$ and the information matrices $\boldsymbol{\Gamma}_{f_{1}}^{(n)}(\theta)$ are defined as if $f_{1}$ is specified, it means that until now the approach is purely parametric, which leads us to consider the semiparametric approach under which $f$ or $f_{1}$ remains completely unspecified is more reasonable. Then, the number of parameters of interest reduced from four to two, the only remaining parameters are therefore, $\beta_{1}$ and $\sigma_{\eta}^{2}$. Redifine $Z_{i j}^{(n)}\left(\beta_{1}\right):=Y_{i j}-\beta_{1} X_{i j}$,

$$
\left.\boldsymbol{\Delta}_{f}^{(n)}\left(\beta_{1}\right):=\left(\begin{array}{c}
\Delta_{f ; 2}^{(n)}\left(\beta_{1}\right) \\
\Delta_{f ; 4}^{(n)}\left(\beta_{1}\right)
\end{array}\right)=\left(\begin{array}{c}
\frac{K_{2}^{(n)}}{\sqrt{n}} \sum_{i=1}^{n} \sum_{j=1}^{m} \phi_{f}\left(Z_{i j}\right) X_{i j} \\
\frac{K_{4}^{(n)}}{2 \sqrt{n}} \sum_{i=1}^{n}\left\{\sum_{j=1}^{m} \psi_{f}\left(Z_{i j}\right) X_{i j}^{2}+\sum_{j=1}^{m} \sum_{\substack{l=1 \\
l \neq j}}^{m} \phi_{f}\left(Z_{i j}\right) \phi_{f}\left(Z_{i l}\right) X_{i j} X_{i l}\right.
\end{array}\right\}\right)
$$

and

$$
\begin{aligned}
& \boldsymbol{\Gamma}_{f}^{(n)}\left(\beta_{1}\right):=\left(\begin{array}{cc}
\Gamma_{f ; 22}^{(n)}\left(\beta_{1}\right) & \Gamma_{f ; 24}^{(n)}\left(\beta_{1}\right) \\
\Gamma_{f ; 24}^{(n)}\left(\beta_{1}\right) & \Gamma_{f ; 44}^{(n)}\left(\beta_{1}\right)
\end{array}\right) \text {, converging, along }(B .3)-\text { subsequences, to } \\
& \boldsymbol{\Gamma}_{f}\left(\beta_{1}\right)=\left(\begin{array}{cc}
m \mathcal{I}_{\phi}(f) & \frac{m \mu_{3}^{X}}{2\left(\mu_{2}^{X} \mu_{4}^{X}\right)^{1 / 2}} \mathcal{I}_{\phi \psi}(f) \\
\frac{m \mu_{3}^{X}}{2\left(\mu_{2}^{X} \mu_{4}^{X}\right)^{1 / 2}} \mathcal{I}_{\phi \psi}(f) & \Gamma_{f ; 44}\left(\beta_{1}\right)
\end{array}\right) \\
& \text { where } \Gamma_{f ; 44}\left(\beta_{1}\right):=\frac{m}{4\left(\mu_{4}^{X}\right)^{1 / 2}}\left\{\mu_{4}^{X}\left(\mathcal{I}_{\psi}(f)-2 \mathcal{I}_{\phi}^{2}(f)\right)+2 m \mathcal{I}_{\phi}^{2}(f) \mu_{2}^{\bar{X}_{\bullet}^{2}}\right\} \text {. }
\end{aligned}
$$

\subsection{Rank-based versions of central sequences}

A general result concerning the relationship between efficient semiparametric procedures and rank-based procedures was established in [14]. In such a context, semiparametrically efficient tests can be obtained by conditioning the $f$-central sequence on the maximal invariant associated with some appropriate generating group.

Denote by, $R_{i j}^{(n)}\left(\beta_{1}\right)$ the rank of the residual $Z_{i j}^{(n)}\left(\beta_{1}\right)$ among the residuals $Z_{11}^{(n)}\left(\beta_{1}\right), \ldots, Z_{n m}^{(n)}\left(\beta_{1}\right)$ and $\mathbf{R}^{(n)}=\mathbf{R}^{(n)}\left(\beta_{1}\right):=\left(R_{11}^{(n)}\left(\beta_{1}\right), \ldots, R_{n m}^{(n)}\left(\beta_{1}\right)\right)$.

From the results of [14], under the null hypothesis $\mathrm{P}_{\beta_{1}, 0 ; f}^{(n)}$, the version of the semiparametrically efficient (at $f$ and $\theta=\left(\beta_{1}, 0\right)$ ) obtained conditioning $\boldsymbol{\Delta}_{f}^{(n)}$ by the rank vector $\mathbf{R}^{(n)}\left(\beta_{1}\right)$

$$
\underline{\Delta}_{f}^{(n)}\left(\beta_{1}\right):=\mathrm{E}\left[\boldsymbol{\Delta}_{f}^{(n)}\left(\beta_{1}\right) \mid \mathbf{R}^{(n)}\left(\beta_{1}\right)\right] .
$$

The conditional definition (4.2) allows us to obtain a statistical test based on the ranks. In practice, this definition of $\boldsymbol{\Delta}_{f}^{(n)}$ (an exact-score) is not appropriate, and the explicit 
approximate-score form (as for the exact-score version, for simplicity using the same notation)

$$
\begin{aligned}
& \underset{\sim}{\Delta_{f}^{(n)}}\left(\beta_{1}\right)=\left(\begin{array}{c}
\Delta_{f ; 2}^{(n)}\left(\beta_{1}\right) \\
\Delta_{f ; 4}^{(n)}\left(\beta_{1}\right)
\end{array}\right) \\
& :=\left(\begin{array}{c}
\frac{\mathcal{K}_{2}^{(n)}}{\sqrt{n}} \sum_{i=1}^{n} \sum_{j=1}^{m}\left[\Phi_{f}\left(\frac{R_{i j}^{(n)}}{N+1}\right)-\bar{\phi}_{f}^{(n)}\right] X_{i j} \\
\left.\frac{K_{4}^{(n)}}{2 \sqrt{n}} \sum_{i=1}^{n}\left\{\sum_{j=1}^{m}\left[\Psi_{f}\left(\frac{R_{i j}^{(n)}}{N+1}\right)-\bar{\psi}_{f}^{(n)}\right] X_{i j}^{2}+\sum_{j=1}^{m} \sum_{\substack{l=1 \\
l \neq j}}^{m}\left[\Phi_{f}\left(\frac{R_{i j}^{(n)}}{N+1}\right)\right) \Phi_{f}\left(\frac{R_{i l}^{(n)}}{N+1}\right)-C_{f}^{(n)}\right] X_{i j} X_{i l}\right\}
\end{array}\right) \\
& =\left(\begin{array}{c}
\frac{\mathcal{K}_{2}^{(n)}}{\sqrt{n}} \sum_{i=1}^{n} \sum_{j=1}^{m} \Phi_{f}\left(\frac{R_{i j}^{(n)}}{N+1}\right)\left[X_{i j}-M_{1}^{(n)}\right] \\
\frac{K_{4}^{(n)}}{2 \sqrt{n}} \sum_{i=1}^{n}\left\{\sum_{j=1}^{m} \Psi_{f}\left(\frac{R_{i j}^{(n)}}{N+1}\right)\left[X_{i j}^{2}-M_{2}^{(n)}\right]+\sum_{j=1}^{m} \sum_{\substack{l=1 \\
l \neq j}}^{m} \Phi_{f}\left(\frac{R_{i j}^{(n)}}{N+1}\right) \Phi_{f}\left(\frac{R_{i l}^{(n)}}{N+1}\right)\left[X_{i j} X_{i l}-C^{(n)}\right]\right\}
\end{array}\right),
\end{aligned}
$$

with $\bar{\phi}_{f}^{(n)}:=\frac{1}{N} \sum_{r=1}^{N} \phi_{f}\left(F^{-1}\left(\frac{r}{N+1}\right)\right), \bar{\psi}_{f}^{(n)}:=\frac{1}{N} \sum_{r=1}^{N} \psi_{f}\left(F^{-1}\left(\frac{r}{N+1}\right)\right)$,

$C_{f}^{(n)}:=\frac{1}{N(N-1)} \sum_{r=1}^{N} \sum_{\substack{s=1 \\ r \neq s}}^{N} \phi_{f}\left(F^{-1}\left(\frac{r}{N+1}\right)\right) \phi_{f}\left(F^{-1}\left(\frac{s}{N+1}\right)\right), \Phi_{f}=\phi_{f} \circ F^{-1}, \Psi_{f}=$ $\psi_{f} \circ F^{-1}$ and $C^{(n)}=\frac{m}{m-1} \overline{\bar{X}}_{\bullet \bullet}^{(n)}-\frac{1}{m-1} M_{1}^{(n)}$.

Let us add the following assumption on $f$, in order to have the equivalence between the approximate- $f$-score rank statistics and the exact- $f$-score rank statistics.

(A.5) The density $f$ is such that $\phi_{f}$ and $\psi_{f}$ are monotones, or the difference between two monotone functions.

Based on Hájek's projection theorem (for more details see, [12]), with $f$ satisfying Assumption (A.5) and under $\mathrm{P}_{\beta_{1}, 0 ; g}^{(n)}$, we have

$$
\begin{aligned}
\underline{\Delta}_{f}^{(n)}\left(\beta_{1}\right)= & \underline{\Delta}_{f}^{(n)}\left(\beta_{1}\right)+o_{\mathrm{P}}(1) \\
:= & \left(\begin{array}{c}
\mathcal{K}_{2}^{(n)} \\
\sqrt{n} \sum_{i=1}^{n} \sum_{j=1}^{m} \Phi_{f}\left(G\left(Z_{i j}^{(n)}\left(\beta_{1}\right)\right)\right)\left[X_{i j}-M_{1}^{(n)}\right] \\
\frac{K_{4}^{(n)}}{2 \sqrt{n}} \sum_{i=1}^{n}\left\{\sum_{j=1}^{m} \Psi_{f}\left(G\left(Z_{i j}^{(n)}\left(\beta_{1}\right)\right)\right)\left[X_{i j}^{2}-M_{2}^{(n)}\right]\right. \\
\left.+\sum_{j=1}^{m} \sum_{\substack{l=1 \\
l \neq j}}^{m} \Phi_{f}\left(G\left(Z_{i j}^{(n)}\left(\beta_{1}\right)\right)\right) \Phi_{f}\left(G\left(Z_{i l}^{(n)}\left(\beta_{1}\right)\right)\right)\left[X_{i j} X_{i l}-C^{(n)}\right]\right\}
\end{array}\right)+o_{\mathrm{P}}(1) .
\end{aligned}
$$

Let Assumptions (A) and (B) hold, under $\mathrm{P}_{\beta_{1}, 0 ; g}^{(n)}, \Delta_{f}^{(n)}\left(\beta_{1}\right)$ is normal with mean zero and covariance matrix

$$
\Gamma_{f}^{(n)}\left(\beta_{1}\right):=\left(\begin{array}{cc}
\Gamma_{f ; 22}^{(n)}\left(\beta_{1}\right) & \Gamma_{f ; 24}^{(n)}\left(\beta_{1}\right) \\
\Gamma_{f ; 24}^{(n)}\left(\beta_{1}\right) & \Gamma_{f ; 44}^{(n)}\left(\beta_{1}\right)
\end{array}\right),
$$

with

$\Gamma_{\sim}^{(n) ; 2}(\theta):=\frac{m\left(M_{2}^{(n)}-\left(M_{1}^{(n)}\right)^{2}\right)}{M_{2}^{(n)}} \mathcal{I}_{\phi}(f), \quad \Gamma_{f ; 24}^{(n)}(\theta):=\frac{m\left(M_{3}^{(n)}-M_{1}^{(n)} M_{2}^{(n)}\right)}{2\left(M_{2}^{(n)} M_{4}^{(n)}\right)^{1 / 2}} \mathcal{I}_{\phi \psi}(f)$, and 


$$
\Gamma_{\sim f ; 44}^{(n)}(\theta):=\frac{1}{4 M_{4}^{(n)}}\left\{m\left(M_{4}^{(n)}-\left(M_{2}^{(n)}\right)^{2}\right) \mathcal{I}_{\psi}(f)+\frac{2}{n} \mathcal{I}_{\phi}^{2}(f) \sum_{i=1}^{n} \sum_{j=1}^{m} \sum_{\substack{l=1 \\ l \neq j}}^{m}\left[X_{i j} X_{i l}-C^{(n)}\right]^{2}\right\} .
$$

\subsection{Semiparametrically optimal rank tests}

The rank based version of $\Delta_{f ; 4}^{*(n)}\left(\beta_{1}\right)$ defined in $(3.2)$ is

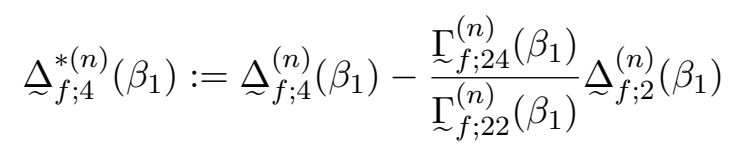

with

$$
\frac{\Gamma_{f ; 24}^{(n)}\left(\beta_{1}\right)}{\Gamma_{f ; 22}^{(n)}\left(\beta_{1}\right)}=\frac{\left(M_{2}^{(n)}\right)^{1 / 2}\left(M_{3}^{(n)}-M_{1}^{(n)} M_{2}^{(n)}\right)}{2\left(M_{4}^{(n)}\right)^{1 / 2}\left(M_{2}^{(n)}-\left(M_{1}^{(n)}\right)^{2}\right)} \frac{\mathcal{I}_{\phi \psi}(f)}{\mathcal{I}_{\phi}(f)}
$$

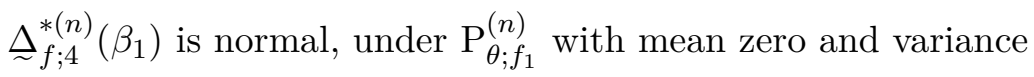

$$
\begin{aligned}
\Gamma_{f ; 4}^{*(n)}\left(\beta_{1}\right) & :=\Gamma_{\sim}^{(n)}\left(\beta_{1}\right)-\frac{\left(\Gamma_{f ; 24}^{(n)}\left(\beta_{1}\right)\right)^{2}}{\Gamma_{f ; 22}^{(n)}\left(\beta_{1}\right)} \\
& =\Gamma_{f ; 44}^{(n)}\left(\beta_{1}\right)-\frac{m}{4} \frac{\left(M_{3}^{(n)}-M_{1}^{(n)} M_{2}^{(n)}\right)^{2}}{M_{4}^{(n)}\left(M_{2}^{(n)}-\left(M_{1}^{(n)}\right)^{2}\right)} \frac{\mathcal{I}_{\phi \psi}^{2}(f)}{\mathcal{I}_{\phi}(f)}
\end{aligned}
$$

Therefore, the test statistic is

$$
{\underset{\sim}{T}}_{f}^{*(n)}\left(\beta_{1}\right):=\left(\Gamma_{\sim}^{\Gamma_{f ; 4}}\left(\beta_{1}\right)\right)^{-1 / 2} \stackrel{\sim}{\Delta}_{f ; 4}^{*(n)}\left(\beta_{1}\right) .
$$

Not that $\beta_{1}$ remains unknown, so it should be replaced by adequate estimator $\hat{\beta}_{1}$. That estimator should be such that both the asymptotic standard normal distribution of $\underset{\sim}{T} T^{*(n)}\left(\hat{\beta}_{1}\right)$ under $\mathrm{P}_{\beta_{1}, 0 ; g}^{(n)}$, and the asymptotic optimality under $\mathrm{P}_{\beta_{1}, 0 ; f}^{(n)}$ of the resulting test be preserved. Thus, let's show that it is possible, but requires a small change in (4.5) indeed in the central sequence $\underset{\sim}{\Delta_{f ; 4}^{*(n)}}\left(\beta_{1}\right)$, that we will then note it by $\underset{\sim}{\stackrel{\bullet}{\bullet} ; 4}\left(\beta_{1}\right)$. Note that this modification only has $o_{\mathrm{P}}(1)$ imapct (the proof will appear later).

$\Delta_{f}^{(n)}\left(\beta_{1}\right)$ is, under $\mathrm{P}_{\beta_{1}, 0 ; g}^{(n)}$, locally asymptotically linear in $\beta_{1}$, that is, satisfies

$$
\begin{aligned}
\Delta_{f}^{(n)}\left(\beta_{1}+n^{-1 / 2} \mathcal{K}_{2}^{(n)} \tau_{2}\right)-\underline{\Delta}_{f}^{(n)}\left(\beta_{1}\right) & =-{\underset{\sim}{\Gamma, g}}_{f,}^{(n)}\left(\beta_{1}\right)\left(\begin{array}{c}
\tau_{2} \\
0
\end{array}\right)+o_{\mathrm{P}}(1) \\
& =-\left(\begin{array}{c}
\Gamma_{f, g ; 22}^{(n)}\left(\beta_{1}\right) \\
\Gamma_{f, g ; 42}^{(n)}\left(\beta_{1}\right)
\end{array}\right) \tau_{2}+o_{\mathrm{P}}(1)
\end{aligned}
$$

with

$$
\begin{aligned}
& \Gamma_{\sim}^{(n)}\left(\beta_{, 22}\left(\beta_{1}\right)=\frac{m\left(M_{2}^{(n)}-\left(M_{1}^{(n)}\right)^{2}\right)}{M_{2}^{(n)}} \mathcal{I}_{\phi}(f, g), \Gamma_{\sim}^{(n)}\left(\beta_{1 ; 42}\right)=\frac{m\left(M_{3}^{(n)}-M_{1}^{(n)} M_{2}^{(n)}\right)}{2\left(M_{2}^{(n)} M_{4}^{(n)}\right)^{1 / 2}} \mathcal{I}_{\psi \phi}(f, g),\right. \\
& \mathcal{I}_{\phi}(f, g):=\int_{0}^{1} \phi_{f}\left(F^{-1}(u)\right) \phi_{g}\left(G^{-1}(u)\right) d u \text { and } \mathcal{I}_{\psi \phi}(f, g):=\int_{0}^{1} \psi_{f}\left(F^{-1}(u)\right) \phi_{g}\left(G^{-1}(u)\right) d u .
\end{aligned}
$$


Note that, for $f=g, \mathcal{I}_{\phi}(f, f)=\mathcal{I}_{\phi}(f) \quad$ and $\quad \mathcal{I}_{\psi \phi}(f, f)=\mathcal{I}_{\psi \phi}(f)$. Then, define

$$
\begin{aligned}
{\underset{\sim}{f ; 4}}_{f(n)}^{\bullet(n)}\left(\beta_{1}\right) & :={\underset{\sim}{f ; 4}}_{f ;}^{(n)}\left(\beta_{1}\right)-\frac{\Gamma_{f, g ; 42}^{(n)}\left(\beta_{1}\right)}{\Gamma_{f, g ; 22}^{(n)}\left(\beta_{1}\right)} \Delta_{f ; 2}^{(n)}\left(\beta_{1}\right) \\
& =\Delta_{f ; 4}^{(n)}\left(\beta_{1}\right)-\frac{\left(M_{2}^{(n)}\right)^{1 / 2}\left(M_{3}^{(n)}-M_{1}^{(n)} M_{2}^{(n)}\right)}{2\left(M_{4}^{(n)}\right)^{1 / 2}\left(M_{2}^{(n)}-\left(M_{1}^{(n)}\right)^{2}\right)} \frac{\mathcal{I}_{\psi \phi}(f, g)}{\mathcal{I}_{\phi}(f, g)} \Delta_{f ; 2}^{(n)}\left(\beta_{1}\right)
\end{aligned}
$$

with variance

$$
\begin{aligned}
\Gamma_{\sim f, g ; 44}^{\bullet(n)}\left(\beta_{1}\right):=\Gamma_{\sim}^{(n)}\left(\beta_{14}\right)-\frac{\left(M_{3}^{(n)}-M_{1}^{(n)} M_{2}^{(n)}\right)^{2}}{4 M_{4}^{(n)}\left(M_{2}^{(n)}-\left(M_{1}^{(n)}\right)^{2}\right)} \frac{\mathcal{I}_{\psi \phi}(f, g)}{\mathcal{I}_{\phi}^{2}(f, g)} \\
\times\left[2 \mathcal{I}_{\phi}(f, g) \mathcal{I}_{\phi \psi}(f)-\mathcal{I}_{\phi}(f) \mathcal{I}_{\psi \phi}(f, g)\right] .
\end{aligned}
$$

While, under $\mathrm{P}_{\beta_{1}, 0 ; g}^{(n)}$ (the $f=g$ case),

$$
\stackrel{\sim}{\sim}_{f ; 4}^{\bullet(n)}\left(\beta_{1}\right)-{\underset{\sim}{f} f ; 4}_{*(n)}^{*(n)}\left(\beta_{1}\right)=\left[-\frac{\Gamma_{\sim}^{(n)} f ; 42}{\Gamma_{f, f ; 22}^{(n)}}+\frac{\Gamma_{f ; 42}^{(n)}}{\Gamma_{f ; 22}^{(n)}}\right] \stackrel{\sim}{f ; 2}_{f ;}^{(n)}\left(\beta_{1}\right)=0 .
$$

Let $\hat{\beta}_{1}^{(n)}$ satisfy Assumption (D.1)-(D.2), it results from asymptotic linearity (4.6) and Lemma 4.4 in [15], under $\mathrm{P}_{\beta_{1}, 0 ; g}^{(n)}$

$$
\begin{aligned}
{\underset{\sim}{\Delta}}_{f ; 4}^{\bullet(n)}\left(\hat{\beta}_{1}^{(n)}\right)-\underset{\sim}{\underset{\sim}{\bullet}} \stackrel{\bullet(n)}{*}\left(\beta_{1}\right) & =\left(-\underset{\sim}{\Gamma_{f, g ; 42}^{(n)}}+\frac{\underset{\sim}{\Gamma_{f, g ; 42}^{(n)}}}{\Gamma_{\sim f, g ; 2}^{(n)}} \Gamma_{f, g ; 22}^{(n)}\right) n^{1 / 2}\left(K_{2}^{(n)}\right)^{-1}\left(\hat{\beta}_{1}^{(n)}-\beta_{1}\right)+o_{\mathrm{P}}(1) \\
& =o_{\mathrm{P}}(1) .
\end{aligned}
$$

The last problem that remains to be solved is that the statistics based on ranks $\underset{\sim}{T_{f}^{\bullet}(n)}\left(\hat{\beta}_{1}^{(n)}\right)$, indeed the variance $\underset{\sim}{\Gamma_{f ; 4}^{\bullet}(n)}\left(\hat{\beta}_{1}^{(n)}\right)$ depends on the cross-information quantities $\mathcal{I}_{\phi}(f, g)$ and $\mathcal{I}_{\psi \phi}(f, g)$ that are unknown and also depends on the unspecified density $g$. We propose here the simplest ones (see [5] and [10])

$$
\begin{aligned}
\widehat{\mathcal{I}}_{\phi ; f}^{(n)}\left(\hat{\beta}_{1}\right):= & \frac{M_{2}^{(n)}}{m c\left(M_{2}^{(n)}-\left(M_{1}^{(n)}\right)^{2}\right)}\left({\underset{\sim}{\Delta ; 2}}_{f ;}^{(n)}\left(\hat{\beta}_{1}\right)-\underset{\sim}{\Delta}(n)\left(\hat{\beta}_{1}+n^{-1 / 2} \mathcal{K}_{2}^{(n)}\right)\right) \\
= & \mathcal{I}_{\phi}(f, g)+o_{\mathrm{P}}(1)
\end{aligned}
$$

and

$$
\begin{aligned}
\widehat{\mathcal{I}}_{\psi \phi ; f}^{(n)}\left(\hat{\beta}_{1}\right) & :=\frac{2\left(M_{2}^{(n)} M_{4}^{(n)}\right)^{1 / 2}}{m d\left(M_{3}^{(n)}-\left(M_{1}^{(n)} M_{2}^{(n)}\right)\right)}\left({ }_{\sim}^{(n)}\left(\hat{\beta}_{1}\right)-\Delta_{f ; 4}^{(n)}\left(\hat{\beta}_{1}+n^{-1 / 2} \mathcal{K}_{2}^{(n)}\right)\right) \\
& =\mathcal{I}_{\psi \phi}(f, g)+o_{\mathrm{P}}(1) .
\end{aligned}
$$

where $c \neq 0$ and $d \neq 0$ are arbitrary constants, whose consistency easily results from asymptotic linearity property (4.6).

We substitute $\mathcal{I}_{\phi}(f, g)$ and $\mathcal{I}_{\psi \phi}(f, g)$ by $\widehat{\mathcal{I}}_{\phi ; f}^{(n)}\left(\hat{\beta}_{1}\right)$ and $\widehat{\mathcal{I}}_{\psi \phi ; f}^{(n)}\left(\hat{\beta}_{1}\right)$, respectively. The rankbased test statistic is $\underset{\sim}{T} \underset{f}{\bullet(n)}\left(\hat{\beta}_{1}^{(n)}\right)$, with

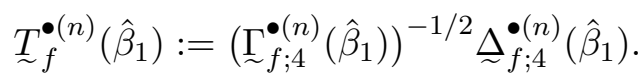


It is easy to verify that the test statistics $\underset{\sim}{T_{f}^{\bullet}}(n)\left(\hat{\beta}_{1}\right)$ and $\underset{\sim}{f_{1}}{ }_{f_{1}}^{(n)}\left(\beta_{1}\right)$ coincide, the following proposition summarizes the results (follows from a straightforward application of Le Cam's third lemma).

Proposition 4.1. Let $\hat{\beta}_{1}^{(n)}$ satisfy Assumptions (D), let Assumptions (B) and $(C)$ hold, fix a density $f$ such that $f_{1} \in \mathcal{F}_{A}$ satisfies (A.5). Then, for any $g_{1} \in \mathcal{F}_{A}$, the sequence of tests rejecting the hypothesis $\bigcup_{g_{1} \in \mathcal{F}_{A}} \mathcal{H}_{0}^{(n)}\left(g_{1}\right)$ whenever $\underset{\sim}{\underset{\sim}{\bullet}(n)}\left(\hat{\beta}_{1}{ }^{(n)}\right)$ exceeds the $(1-\alpha)$ standard normal quantile $z_{\alpha}$

(i) has asymptotic level $\alpha$,

(ii) is, along (B.3)-subsequences, semiparametrically locally asymptotically most powerful unbiased, at asymptotic level $\alpha$, against alternatives of the form

$\bigcup_{\beta_{1}} \bigcup_{\sigma_{\eta}^{2}>0} \bigcup_{h_{1} \in \mathcal{F}_{C \mid f_{1}}}\left\{\mathrm{P}_{\beta_{1}, \sigma_{\eta}^{2} ; f_{1}, h_{1}}^{(n)}\right\}$.

(iii) still along (B.3)-subsequences, this test has asymptotic power

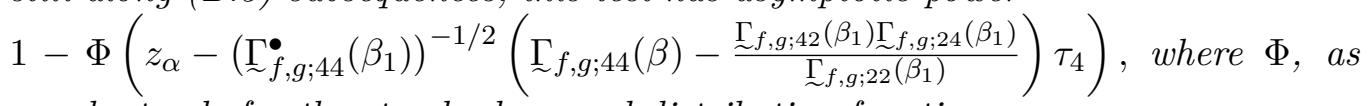
usual, stands for the standard normal distribution function, against $\mathrm{P}_{\beta_{1}, n^{-1 / 2} K_{4}^{(n)} \tau_{4} ; g_{1}, h_{1}}^{(n)}$.

\subsection{The Wilcoxon and van der Waerden test statistics}

Particular cases most importantly are the van der Waerden and the Wilcoxon, which are optimal under normal and logistic densities, respectively.

- The van der Waerden test statistic (normal scores): given for $f=\phi$, where $\psi_{f}\left(F^{-1}(u)\right)=\left(\Phi^{-1}(u)\right)^{2}-1$ and $\phi_{f}\left(F^{-1}(u)\right)=\Phi^{-1}(u)$ takes the form:

$$
\begin{aligned}
\underset{\sim}{T_{v d W}^{\bullet(n)}\left(\beta_{1}\right)}: & =\frac{n^{-1 / 2}}{s_{v d W}^{(n)}}\left\{\sum_{i=1}^{n} \sum_{j=1}^{m}\left(\Phi^{-1}\left(\frac{R_{i j}^{(n)}}{N+1}\right)\right)^{2}\left[X_{i j}^{2}-M_{2}^{(n)}\right]\right. \\
& -\frac{\left(M_{3}^{(n)}-M_{1}^{(n)} M_{2}^{(n)}\right) \widehat{\mathcal{I}}_{\psi \phi ; v d W}}{\left(M_{2}^{(n)}-\left(M_{1}^{(n)}\right)^{2}\right) \widehat{\mathcal{I}}_{\phi ; v d W}} \sum_{i=1}^{m} \sum_{j=1}^{m} \Phi^{-1}\left(\frac{R_{i j}^{(n)}}{N+1}\right)\left[X_{i j}-M_{1}^{(n)}\right] \\
& \left.+\sum_{i=1}^{n} \sum_{j=1}^{m} \sum_{\substack{l=1 \\
l \neq j}}^{m} \Phi^{-1}\left(\frac{R_{i j}^{(n)}}{N+1}\right) \Phi^{-1}\left(\frac{R_{i l}^{(n)}}{N+1}\right)\left[X_{i j} X_{i l}-C^{(n)}\right]\right\}
\end{aligned}
$$

with

$$
\begin{aligned}
\left(s_{v d W}^{(n)}\right)^{2}=2 m\left(M_{4}^{(n)}\right. & \left.-\left(M_{2}^{(n)}\right)^{2}\right)+m \frac{\left(M_{3}^{(n)}-M_{1}^{(n)} M_{2}^{(n)}\right)}{\left(M_{2}^{(n)}-\left(M_{1}^{(n)}\right)^{2}\right)}\left(\frac{\widehat{\mathcal{I}}_{\psi \phi ; v d W}}{\widehat{\mathcal{I}}_{\phi ; v d W}}\right)^{2} \\
& +2 m\left(m \overline{\bar{X}}_{\bullet \bullet}^{(n) 2}-M_{4}^{(n)}-(m-1) C^{(n) 2}\right)
\end{aligned}
$$

where $\widehat{\mathcal{I}}_{\phi ; v d W}$ and $\widehat{\mathcal{I}}_{\psi \phi ; v d W}$ stand for $\widehat{\mathcal{I}}_{\phi ; f}\left(\hat{\beta}_{1}\right)$ and $\widehat{\mathcal{I}}_{\psi \phi ; f}\left(\hat{\beta}_{1}\right)$ respectively $(f$ the normal density).

- The Wilcoxon test statistic (logistic scores): given for $f=\ell$, where $\psi_{f}\left(F^{-1}(u)\right)=$ $6 u(u-1)+1$ and $\phi_{f}\left(F^{-1}(u)\right)=2 u-1$ takes the form: 


$$
\begin{aligned}
{\underset{\sim}{T}}_{W}^{\bullet(n)}\left(\beta_{1}\right) & :=\frac{n^{-1 / 2}}{s_{W}^{(n)}}\left\{3 \sum_{i=1}^{n} \sum_{j=1}^{m}\left(\frac{R_{i j}^{(n)}}{N+1}\right)\left(\frac{R_{i j}^{(n)}}{N+1}-1\right)\left[X_{i j}^{2}-M_{2}^{(n)}\right]\right. \\
& -\frac{\left(M_{3}^{(n)}-M_{1}^{(n)} M_{2}^{(n)}\right) \widehat{\mathcal{I}}_{\psi \phi ; v d W}}{\left(M_{2}^{(n)}-\left(M_{1}^{(n)}\right)^{2}\right) \widehat{\mathcal{I}}_{\phi ; v d W}} \sum_{i=1}^{m}\left(\frac{R_{i j}^{(n)}}{N+1}\right)\left[X_{i j}-M_{1}^{(n)}\right] \\
& \left.+2 \sum_{i=1}^{n} \sum_{j=1}^{m} \sum_{\substack{l=1 \\
l \neq j}}^{m}\left(\frac{R_{i j}^{(n)}}{N+1}\right)\left(\frac{R_{i l}^{(n)}}{N+1}\right)\left[X_{i j} X_{i l}-C^{(n)}\right]\right\}
\end{aligned}
$$

with

$$
\begin{aligned}
\left(s_{W}^{(n)}\right)^{2}=\frac{m}{20}\left(M_{4}^{(n)}\right. & \left.-\left(M_{2}^{(n)}\right)^{2}\right)+\frac{m}{12} \frac{\left(M_{3}^{(n)}-M_{1}^{(n)} M_{2}^{(n)}\right)}{\left(M_{2}^{(n)}-\left(M_{1}^{(n)}\right)^{2}\right)}\left(\frac{\widehat{\mathcal{I}}_{\psi \phi ; v d W}}{\widehat{\mathcal{I}}_{\phi ; v d W}}\right)^{2} \\
& +\frac{m}{18}\left(m \overline{\bar{X}}_{\bullet \bullet}^{(n) 2}-M_{4}^{(n)}-(m-1) C^{(n) 2}\right)
\end{aligned}
$$

where $\widehat{\mathcal{I}}_{\phi ; W}$ and $\widehat{\mathcal{I}}_{\psi \phi ; W}$ stand for $\widehat{\mathcal{I}}_{\phi ; f}\left(\hat{\beta}_{1}\right)$ and $\widehat{\mathcal{I}}_{\psi \phi ; f}\left(\hat{\beta}_{1}\right)$ respectively $(f$ the logistic density).

\section{Simulation}

The objective of this section is to evaluate the performance of the proposed tests at the asymptotic level $\alpha=5 \%$. Using R-programming, we consider a simulation of $N=2500$ independent samples of size $n m=100 * 5$ from the model:

$$
Y_{i j}=\beta_{0}+\left(\beta_{1}+\eta_{i}\right) X_{i j}+\varepsilon_{i j}, \quad i=1, \ldots, 100, \quad j=1, \ldots, 5,
$$

where

(a) $\beta_{0}=1$ and $\beta_{1}=10$,

(b) the $X_{i j}$ 's are i.i.d. normal $(0,1)$,

(c) the $\eta_{i}$ 's are i.i.d. Gaussian with mean zero and standard deviation $\sigma_{\eta}=0$ (for null hypothesis), $=0.1,0.15,0.2,0.25$ or 0.3 (for increasing alternatives),

(d) the $\varepsilon_{i j}$ 's are i.i.d. with symmetric densities: Gaussian $\left(\phi_{1}\right), \operatorname{logistic}\left(\ell_{1}\right), \operatorname{student}\left(t_{5}\right)$; with asymmetric densities: skew normal $(s \mathcal{N})$, skew Student $t_{5}\left(s t_{5}\right)^{\dagger}$ (both with skewness parameter value $\delta=3$ ).

For each replication, we performed the following tests at the asymptotic level $\alpha=5 \%$ : The Likelihood Ratio Test $(L R T)$ on (1.3), the square of Gaussian test based on $T_{\phi_{1}}^{*(n)}$, the pseudo-Gaussian test based on $T_{\phi_{1}}^{\bullet(n)}$, the van der Waerden test based on $\underset{\sim}{T}(n)$, , the Wilcoxon test based on $\underset{\sim}{T}(n)$ and the rank tests based on Student scores with 5 degrees of freedom $\underset{\sim}{T_{5}}(n)$. Rejection frequencies are reported in Table 1 .

These simulations shows that the Likelihood Ratio test and the square of Gaussian test $T_{\phi_{1}}^{*(n)}$ coincides. It shows also that the pseudo-Gaussian test $T_{\phi_{1}}^{\bullet(n)}$ confirms the good overall performance. More under asymmetric densities (skew normal and skew-Student), it shows the superiority of ranking tests over pseudo-Gaussian tests.

$\dagger_{\text {for a definition }}$ of skew-normal and skew-Student densities. See, for details, [2] 
Table 1. Rejection frequencies (out of 2500 replications), for $\sigma_{\eta}=0$ (null hypothesis) and various non-zero values of $\sigma_{\eta}$ (local alternative hypothesis), with error distribution that is normal $\left(\phi_{1}\right)$, logistic $\left(\ell_{1}\right)$, Student $\left(t_{5}\right)$, skew-normal $(s \mathcal{N}(3))$ and skew-Student $\left(s t_{5}(3)\right)$ of Likelihood Ratio test, square of the Gaussian test, the pseudo-Gaussian test, the van der Waerden test, the Wilcoxon test. The sample size is $500(n=100$ and $m=5)$.

\begin{tabular}{|c|c|c|c|c|c|c|c|}
\hline \multirow[t]{2}{*}{$g_{1}$} & \multirow[t]{2}{*}{ Test } & \multicolumn{6}{|c|}{$\sigma_{\eta}$} \\
\hline & & 0 & 0.1 & 0.15 & 0.2 & 0.25 & 0.3 \\
\hline \multirow{6}{*}{$\phi_{1}$} & $L R T$ & .0488 & 0968 & 0.1848 & 0.3720 & 0.5780 & 0.7928 \\
\hline & $\left(T_{\phi_{1}}^{(n) \bullet}\right)^{2}$ & 0.0408 & .0924 & 0.1776 & 0.3680 & 0.5884 & 0.7980 \\
\hline & $T_{\phi_{1}}^{(n) \bullet}$ & 0.0548 & 0.1276 & 0.2376 & 0.4328 & 0.6504 & 0.8472 \\
\hline & $T_{v d W}^{(n)}$ & 0.0584 & 0.1588 & 0.2636 & 0.4516 & 0.6624 & 0.8576 \\
\hline & $T_{W}^{(n)}$ & 0.0528 & 0.1424 & 0.2456 & 0.4324 & 0.6460 & 0.8184 \\
\hline & $T_{t_{5}}^{(n)}$ & 0516 & 0.1456 & 0.2620 & 0.4196 & 0.5960 & 0.7904 \\
\hline \multirow{6}{*}{$l_{1}$} & "LRT & 480 & 1000 & 0.1592 & 0.3080 & 0.4960 & $\overline{0.6960}$ \\
\hline & $\left(T_{\phi_{1}}^{(n) \bullet}\right)^{2}$ & .0420 & .1080 & 0.1620 & 0.3112 & 0.5160 & 0.7040 \\
\hline & $T_{\phi_{1}}^{(n) \bullet}$ & 0.0524 & 0.1340 & 0.2324 & 0.4252 & 0.6348 & 0.8204 \\
\hline & $T_{v d W}^{(n)}$ & 0520 & 0.1344 & 0.2352 & 0.4328 & 0.6420 & 0.8252 \\
\hline & $T_{W}^{(n)}$ & 0.0560 & 0.1544 & 0.2904 & 0.5016 & 0.6984 & 0.8720 \\
\hline & $T_{t_{5}}^{(n)}$ & .0532 & 0.1492 & 0.2840 & 0.4808 & 0.6996 & 0.8692 \\
\hline \multirow{6}{*}{$t_{5}$} & 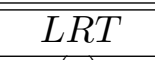 & .0480 & 0.1092 & 0.1960 & 0.3600 & (0.5680 & 0.7400 \\
\hline & $\left(T_{\phi_{1}}^{(n) \bullet}\right)^{2}$ & 0.0492 & 0.0922 & 0.1860 & 0.3360 & 0.5560 & 0.7440 \\
\hline & $T_{\phi_{1}}^{(n) \bullet}$ & 0.0512 & 0.1596 & 0.2864 & 0.4756 & 0.6736 & 0.8464 \\
\hline & $T_{v d W}^{(n)}$ & .0532 & 0.1684 & 0.3296 & 0.5216 & 0.7196 & 0.8932 \\
\hline & $T_{W}^{(n)}$ & & 0.1808 & 0.3164 & 0.5444 & 0.7520 & 0.9084 \\
\hline & $T_{t_{5}}^{(n)}$ & 0572 & 0.1916 & 0.3620 & 0.5604 & 0.7932 & 0.9492 \\
\hline \multirow{6}{*}{$s \mathcal{N}(3)$} & LRT & .0480 & 0.1040 & 0.2020 & 0.3916 & 0.6004 & 0.7764 \\
\hline & $\left(T_{\phi_{1}}^{(n) \bullet}\right)^{2}$ & 0.0424 & 0.1000 & 0.1960 & 0.3660 & 0.5976 & 0.7640 \\
\hline & $T_{\phi_{1}}^{(n) \bullet}$ & 0.0512 & 0.1832 & 0.3372 & 0.5388 & 0.7492 & 0.8912 \\
\hline & $T_{v d W}^{(n)}$ & 0.0576 & 0.1932 & 0.3556 & 0.5920 & 0.8048 & 0.9324 \\
\hline & $T_{W}^{(n)}$ & 0.0564 & 0.1968 & 0.3456 & 0.5492 & 0.7620 & 0.9196 \\
\hline & $T_{t_{5}}^{(n)}$ & 0528 & 0.1692 & 0.3028 & 0.4916 & 0.7232 & 0.8612 \\
\hline \multirow{6}{*}{$s t_{5}(3)$} & LRT & 0.0484 & 0.1164 & 0.2040 & 0.3464 & 0.5364 & 0.7328 \\
\hline & $\left(T_{\phi_{1}}^{(n) \bullet}\right)^{2}$ & 0.0496 & 0.1288 & 0.2120 & 0.3532 & 0.5404 & 0.7324 \\
\hline & $T_{\phi_{1}}^{(n) \bullet}$ & 0.0504 & 0.1656 & 0.2856 & 0.4856 & 0.6932 & 0.8664 \\
\hline & $T_{v d W}^{(n)}$ & 0.0584 & 0.2348 & 0.4896 & 0.6824 & 0.8932 & 0.9692 \\
\hline & $T_{W}^{(n)}$ & 0.0524 & 0.2116 & 0.4192 & 0.6428 & 0.8440 & 0.9508 \\
\hline & $T_{t_{5}}^{(n)}$ & 0.0516 & 0.2064 & 0.4012 & 0.6244 & 0.8252 & 0.9436 \\
\hline
\end{tabular}

\section{Real data analysis}

In this section, we illustrate an application of the usual likelihood ratio test (LRT), the pseudo-Gaussian test $T_{\phi_{1}}^{\bullet(n)}$, and the van der Waerden test $\underset{\sim}{T_{v d W}^{\bullet(n)}}$ in a real dataset. Our 
study is related to a growth curve (Longitudinal data, where individuals are repeatedly measured over time) problem from dentistry. The original data set is from ([23]) available, under the name "Orthodont", in the packages lme4 and nlme in R ([3,22]). The Orthodont data has measurement on the distance (y) (in millimeters) between two positions on the skull (the center of the pituitary and the pterygomaxillary fissure), taken every two years from 8 until age $(\mathrm{x}) 14$ (i.e. at the ages $8,10,12$, and 14), on 16 males and 11 females. This distance was measured four times for each of the 27 subjects (individual children). In this example, we are interest on the random slope for the simple linear mixed model with equal intercepts. Consider here:

$$
y_{i j}=\beta_{0}+\left(\beta_{1}+\eta_{i}\right) x_{i j}+\varepsilon_{i j} \text { for } i=1, \ldots, 27 \text { and } j=1, \ldots, 4
$$

with

- $\beta_{0}$ and $\beta_{1}$ are, respectively, the fixed effects for the intercept and the slope,

- $\varepsilon_{i j} \sim \mathcal{N}\left(0, \sigma^{2}\right)$,

- $\eta_{i} \sim \mathcal{N}\left(0, \sigma_{\eta}^{2}\right)$.

We will use the likelihood ratio test (LRT), the pseudo-Gaussian test $T_{\phi_{1}}^{\bullet(n)}$, and the van der Waerden test $\underset{\sim}{T_{v d W}^{\bullet}(n)}$ to test the null hypothesis $\mathcal{H}_{0}: \sigma_{\eta}^{2}=0$, versus the alternative $\mathcal{H}_{1}: \sigma_{\eta}^{2}>0$, comparing a fitted full-model (6.1) with the parameter of interest (i.e: random-slope) having $\left(p_{1}=4\right)$ estimable parameters and a fitted model having $\left(p_{2}=3\right)$ estimable parameters without the parameter of interest (i.e.: reduced model, without random-slope)

$$
y_{i j}=\beta_{0}+\beta_{1} x_{i j}+\varepsilon_{i j} \text { for } i=1, \ldots, 27 \text { and } j=1, \ldots, 4 .
$$

Note that if the calculated value of LRT, $T_{\phi_{1}}^{\bullet(n)}$, and $\underset{\sim}{T_{v d W}^{\bullet(n)}}$ is larger than the critical value of the chi-squared distribution with $\left(p_{1}-p_{2}=1\right)$ degrees of freedom, and the normal distribution respectively, the parameter of interest should be retained in the model. At the usual significance level $(\alpha=5 \%)$, the following table presents the result:

\begin{tabular}{|c||c|c|}
\hline Test & Calculated value & critical value \\
\hline LRT & 64.08381 & $\chi^{2}(1)=3.84$ \\
\hline$T_{\phi_{1}}^{\bullet(n)}$ & 8.112118 & $Z_{\alpha}=1.644854$ \\
\hline$T_{v d W}^{\bullet(n)}$ & 8.352332 & $Z_{\alpha}=1.644854$ \\
\hline
\end{tabular}

The observed values of the statistical tests are $\mathrm{LRT}=64.08381\left(\mathrm{p}\right.$-value $\left.=1.192 \times 10^{-15}\right)$, $T_{\phi_{1}}^{\bullet(n)}=8.112118\left(\mathrm{p}\right.$-value $\left.2.487 \times 10^{-16}\right)$ and ${\underset{\sim}{v}}_{v d W}^{\bullet(n)}=8.352332\left(\mathrm{p}\right.$-value $\left.=3.346 \times 10^{-17}\right)$, shows that the three tests lead to the same conclusion, namely that $\mathcal{H}_{0}$ should be rejected at the usual significance levels. The results suggest the proposed test appears to be more powerful than LR test, for detecting the random slope. This conclusion agrees with the results of the permutation tests, described in [8] and applied in the orthodontic data, for testing the intercept and/or slope random effects in linear growth curve model. Recall that, the permutation test is obtained by conditioning with respect to the order statistic. Whereas invariance arguments lead to rank-based tests, unbiasedness arguments thus lead to permutation tests. In choosing between the two tests, we prefer rank test for detecting the random slope.

\section{Conclusion}

For testing randomness in the linear mixed models, we propose a test statistics valid for a large class of densities rather than the likelihood ratio test which is restricted to the Gaussian one. Those tests are constructed using Le Cam methodology, their performance 
are remarkably high compared to the conventional ones.

The simulations, based on the rejection frequency, for different tests, guarantee the good performance of the proposed tests. It also appears that skewed and heavy-tailed densities signficantly improves the superiority of rank tests over the Likelihood ratio procedure. A real example of longitudinal data is used to illustrate the behavior of the new test.

\section{Acknowledgements}

The authors would like to express their gratitude to the two referees and the editor for their constructive comments and suggestions which improved the presentation of the paper. We would also like to thank professor Aicha Bouchara for her linguistic correction.

\section{References}

[1] A. Akharif and M. Hallin, Efficient detection of random coefficients in autoregressive models, Ann. Statist. 31 (2), 675-704, 2003.

[2] A. Azzalini and A. Capitanio, Distributions generated by perturbation of symmetry with emphasis on a multivariate skew t-distribution, J. R. Stat. Soc. Ser. B. Stat. Methodol. 65 (2), 367-389, 2003.

[3] D.M. Bates, M. Machler, B. Bolker and S. Walker, Fitting linear mixed effects models using lme4, J. Stat. Softw. 67 (1), 1-48, 2015.

[4] N. Bennala, M. Hallin and D. Paindaveine, Pseudo-Gaussian and rank-based optimal tests for random individual effects in large $n$ small t panels, J. Econometrics 170 (1), 50-67, 2012.

[5] C. Delphine, M. Hallin and D. Paindaveine, On the estimation of cross-information quantities in rank-based inference, in Nonparametrics and Robustness in Modern Statistical Inference and Time Series Analysis: A Festschrift in Honor of Professor Jana Jurečkovà, Institute of Mathematical Statistics, 7, 35-45, 2010.

[6] E. Demidenko, Mixed Models: Theory and Applications with R, John Wiley and Sons, 2013.

[7] R. Drikvandi and S. Noorian, Testing random effects in linear mixed-effects models with serially correlated errors, Biom. J. 61 (4), 802-812, 2019.

[8] R. Drikvandi, A. Khodadadi and G. Verbeke, Testing variance components in balanced linear growth curve models, J. Appl. Stat. 39 (3), 563-572, 2012.

[9] J.J. Droesbeke and J. Fine, Inférence non paramétrique, Les statistiques de rangs, Éditions de l'Université de Bruxelles, 1996.

[10] M. Fihri, A. Akharif, A. Mellouk and M. Hallin, Efficient pseudo-Gaussian and rankbased detection of random regression coefficients, J. Nonparametr. Stat. 32 (2), 367402, 2020.

[11] J.L. Foulley, C. Delmas and C. Robert-Granié, Méthodes du maximum de vraisemblance en modèle linéaire mixte, J Soc Stat Paris 143 (1-2), 5-52, 2002.

[12] J. Hájek and Z. Šidák, Theory of Rank Tests, Academic Press, New York, 1967.

[13] J. Hájek, Local asymptotic minimax and admissibility in estimation, in: Proceedings of the Sixth Berkeley Symposium on Mathematical Statistics and Probability, Volume 1: Theory of Statistics, Berkeley, CA: University of California Press, 1972.

[14] M. Hallin and B.J.M. Werker, Semiparametric efficiency, distribution-freeness and invariance, Bernoulli 9 (1), 137-165, 2003.

[15] J.P. Kreiss, On adaptive estimation in stationary ARMA processes, Ann. Statist. 15 (1), 112-133, 1987.

[16] N.M. Laird and J.H. Ware, Random-effects models for longitudinal data, Biometrics 38 (4), 963-974, 1982. 
[17] L. Le Cam, Asymptotic Methods in Statistical Decision Theory, Springer-Verlag, New York, 1986.

[18] L.M. Le Cam and G.L. Yang, Asymptotics in Statistics: Some Basic Concepts, 2 edn, Springer-Verlag, New York, 2000.

[19] A. Lmakri, A. Akharif and A. Mellouk, Optimal detection of bilinear dependence in short panels of regression data, Rev. Colomb. Estad. 43 (2), 143-171, 2020.

[20] C.H. Morrell, Likelihood ratio testing of variance components in the linear mixedeffects model using restricted maximum likelihood, Biometrics 54 (4), 1560-1568, 1998.

[21] G.E. Noether, On a theorem by wald and wolfowitz, Ann. Math. Statist. 20 (3), 455458, 1949.

[22] J. Pinheiro and D. Bates, Mixed-Effects Models in S and S-PLUS, Springer Science and Business Media, 2006.

[23] R.F. Potthoff and S.N. Roy, A generalized multivariate analysis of variance model useful especially for growth curve problems, Biometrika 51 (3-4), 313-326, 1964.

[24] K. Rao, R. Drikvandi and B. Saville, Permutation and Bayesian tests for testing random effects in linear mixed-effects models, Stat. Med. 38 (25), 5034-5047, 2019.

[25] M. Regis, A. Brini, N. Nooraee, R. Haakma and E.R. van den Heuvel, The linear mixed model: model formulation, identifiability and estimation, Comm. Statist. Simulation Comput. Doi:10.1080/03610918.2019.1694153, 2019.

[26] A.R. Swensen, The asymptotic distribution of the likelihood ratio for autoregressive time series with a regression trend, J. Multivariate Anal. 16 (1), 54-70, 1985.

[27] G. Verbeke, Linear Mixed Models for Longitudinal Data, Linear Mixed Models in Practice, Springer, New York, 63-153, 1997.

\section{Appendix A. Proof of Proposition 2.1}

The proof of 2.1 is to ensure that the six conditions (Conditions 1.2 to 1.7) in Lemma 1 [26] are satisfied, the only delicate one actually is condition (1.2). This condition is a direct result (see Lemma 2 Swensen) of the quadratic mean differentiability in the neighborhood of any $\left(\beta_{0}, \beta_{1}, \sigma^{2}, 0\right)$.

$$
\left(\beta_{0}, \beta_{1}, \sigma^{2}, \sigma_{\eta}^{2}\right) \longmapsto q_{\beta_{0}, \beta_{1}, \sigma^{2}, \sigma_{\eta}^{2} ; f_{1}}^{1 / 2}(y):=\left\{\frac{1}{\sigma^{m}} \int_{\mathbb{R}} \prod_{j=1}^{m} f_{1}\left(\frac{1}{\sigma}\left(y_{j}-\beta_{0}-\beta_{1} x_{j}-\sigma_{\eta} \eta x_{j}\right)\right) h(\eta) d \eta\right\}^{1 / 2},
$$

with $y=\left(y_{1}, y_{2}, \ldots, y_{m}\right)^{\prime} \in \mathbb{R}^{m}$.

Quadratic mean differentiability is established in the following lemma.

Lemma A.1. Let assumptions $(B)$ and $(C)$ hold and fix $f_{1} \in \mathcal{F}_{A}$. Define, for $y \in \mathbb{R}^{m}$,

$$
\begin{aligned}
& D_{\beta_{0}} q_{\beta_{0}, \beta_{1}, \sigma^{2}, 0 ; f_{1}}^{1 / 2}(y):=\frac{1}{2 \sigma} q_{\beta_{0}, \beta_{1}, \sigma^{2}, 0 ; f_{1}}^{1 / 2}(y) \sum_{j=1}^{m} \phi_{f_{1}}\left(\frac{y_{j}-\beta_{0}-\beta_{1} x_{j}}{\sigma}\right), \\
& D_{\beta_{1}} q_{\beta_{0}, \beta_{1}, \sigma^{2}, 0 ; f_{1}}^{1 / 2}(y):=\frac{1}{2 \sigma} q_{\beta_{0}, \beta_{1}, \sigma^{2}, 0 ; f_{1}}^{1 / 2}(y) \sum_{j=1}^{m} \phi_{f_{1}}\left(\frac{y_{j}-\beta_{0}-\beta_{1} x_{j}}{\sigma}\right) K_{1}^{(n)} x_{j}, \\
& D_{\sigma^{2}} q_{\beta_{0}, \beta_{1}, \sigma^{2}, 0 ; f_{1}}^{1 / 2}(y):=\frac{1}{2 \sigma} q_{\beta_{0}, \beta_{1}, \sigma^{2}, 0 ; f_{1}}^{1 / 2}(y) \sum_{j=1}^{m}\left[\left(\frac{y_{j}-\beta_{0}-\beta_{1} x_{j}}{\sigma}\right) \phi_{f_{1}}\left(\frac{y_{j}-\beta_{0}-\beta_{1} x_{j}}{\sigma}\right)-1\right],
\end{aligned}
$$

and

$$
\begin{array}{r}
D_{\sigma_{\eta}^{2}} q_{\beta_{0}, \beta_{1}, \sigma^{2}, \sigma_{\eta}^{2} ; f_{1}}^{1 / 2}(y)_{\mid \sigma_{\eta}^{2}=0}:=\frac{1}{4 \sigma^{2}} q_{\beta_{0}, \beta_{1}, \sigma^{2}, 0 ; f_{1}}^{1 / 2}(y)\left[\sum_{j=1}^{m} \psi_{f_{1}}\left(\frac{y_{j}-\beta_{0}-\beta_{1} x_{j}}{\sigma}\right) x_{j}^{2}\right. \\
\left.+\sum_{j=1}^{m} \sum_{\substack{l=1 \\
l \neq j}}^{m} \phi_{f_{1}}\left(\frac{y_{j}-\beta_{0}-\beta_{1} x_{j}}{\sigma}\right) \phi_{f_{1}}\left(\frac{y_{l}-\beta_{0}-\beta_{1} x_{l}}{\sigma}\right) x_{j} x_{l}\right] .
\end{array}
$$


Then as $t, s, v$, and $r \rightarrow 0$,

(i) $\int\left[q_{\beta_{0}+t, \beta_{1}+s, \sigma^{2}+v, r^{2} ; f_{1}}^{1 / 2}(y)-q_{\beta_{0}+t, \beta_{1}+s, \sigma^{2}+v, 0 ; f_{1}}^{1 / 2}(y)-r^{2} D_{\sigma_{\eta}^{2}} q_{\beta_{0}+t, \beta+s, \sigma^{2}+v, \sigma_{\eta}^{2} ; f_{1}}^{1 / 2}(y)_{\mid \sigma_{\eta}^{2}=0}\right]^{2} d y=o\left(r^{4}\right)$,

(ii) $\int\left[q_{\beta_{0}+t, \beta_{1}+s, \sigma^{2}+v, 0 ; f_{1}}^{1 / 2}(y)-q_{\beta_{0}, \beta_{1}, \sigma^{2}, 0 ; f_{1}}^{1 / 2}(y)-(t, s, v)\left(\begin{array}{c}D_{\beta_{0}} q_{\beta_{0}, \beta_{1}, \sigma^{2}, 0 ; f_{1}}^{1 / 2}(y) \\ D_{\beta_{1}} q_{\beta_{0}, \beta_{1}, \sigma^{2}, j_{1} f_{1}}^{1 / y)} \\ D_{\sigma^{2}} q_{\beta_{0}, \beta_{1}, \sigma^{2}, 0 ; f_{1}}^{1 / 2}(y)\end{array}\right)\right]^{2} d y=o\left(\left\|(t, s, v)^{\prime}\right\|^{2}\right)$,

(iii) $\int\left[D_{\sigma_{\eta}^{2}} q_{\beta_{0}+t, \beta_{1}+s, \sigma^{2}+v, \sigma_{\eta}^{2} ; f_{1}}^{1 / 2}(y)_{\mid \sigma_{\eta}^{2}=0}-D_{\sigma_{\eta}^{2}} q_{\beta_{0}, \beta_{1}, \sigma^{2}, \sigma_{\eta}^{2} ; f_{1}}^{1 / 2}(y)_{\mid \sigma_{\eta}^{2}=0}\right]^{2} d y=o(1) \quad$ and

(iv) $\int\left[q_{\beta_{0}+t, \beta_{1}+s, \sigma^{2}+v, r^{2} ; f_{1}}^{1 / 2}(y)-q_{\beta_{0}, \beta_{1}, \sigma^{2}, 0 ; f_{1}}^{1 / 2}(y)-\left(t, s, v, r^{2}\right)\left(\begin{array}{c}D_{\beta_{0}} q_{\beta_{0}, \beta_{1}, \sigma^{2}, 0 ; f_{1}}^{1 / 2}(y) \\ D_{\beta_{1}} q_{\beta_{0}, \beta_{1}, \sigma^{2}, 0 ; f_{1}}(y) \\ D_{\sigma^{2}} q_{\beta_{0}, \beta_{1}, \sigma^{2}, 0 ; f_{1}}^{1 / 2}(y) \\ D_{\sigma_{\eta}^{2}}^{1 / 2} q_{\beta_{0}, \beta_{1}, \sigma^{2}, \sigma_{\eta}^{2} ; f_{1}}(y)_{\mid \sigma_{\eta}^{2}=0}\end{array}\right)\right]^{2} d y=o\left(\left\|\left(t, s, v, r^{2}\right)^{\prime}\right\|^{2}\right)$.

Proof. (i) Let $z_{j}=y_{j}-\left(\beta_{0}+t\right)-\left(\beta_{1}+s\right) x_{j}$ and $\mathbf{z}:=\left(z_{1}, z_{2}, \ldots, z_{m}\right)^{\prime}$, the left part of point (i) shall take the following form:

$$
\begin{aligned}
\int_{\mathbb{R}^{m}} & {\left[\left\{\frac{1}{\left(\sigma^{2}+v\right)^{m / 2}} \int_{\mathbb{R}_{j=1}}^{m} f_{1}\left(\frac{z_{j}-r \eta x_{j}}{\left(\sigma^{2}+v\right)^{1 / 2}}\right) h(\eta) d \eta\right\}^{1 / 2}-\left\{\frac{1}{\left(\sigma^{2}+v\right)^{m / 2}} \prod_{j=1}^{m} f_{1}\left(\frac{z_{j}}{\left(\sigma^{2}+v\right)^{1 / 2}}\right)\right\}^{1 / 2}\right.} \\
- & \frac{r^{2}}{4\left(\sigma^{2}+v\right)}\left\{\frac{1}{\left(\sigma^{2}+v\right)^{m / 2}} \prod_{j=1}^{m} f_{1}\left(\frac{z_{j}}{\left(\sigma^{2}+v\right)^{1 / 2}}\right)\right\}^{1 / 2} \\
& \left.\times\left\{\sum_{j=1}^{m} \psi_{f_{1}}\left(\frac{z_{j}}{\left(\sigma^{2}+v\right)^{1 / 2}}\right) x_{j}^{2}+\sum_{j=1}^{m} \sum_{\substack{l=1 \\
l \neq j}}^{m} \phi_{f_{1}}\left(\frac{z_{j}}{\left(\sigma^{2}+v\right)^{1 / 2}}\right) \phi_{f_{1}}\left(\frac{z_{l}}{\left(\sigma^{2}+v\right)^{1 / 2}}\right) x_{j} x_{l}\right\}\right]^{2} d \mathbf{z}
\end{aligned}
$$

which is equivalent to

$$
\begin{aligned}
\int_{\mathbb{R}^{m}}\left[\left\{\int_{\mathbb{R}} \prod_{j=1}^{m} f\left(z_{j}-r \eta x_{j}\right) h(\eta) d \eta\right\}^{1 / 2}-\left\{\prod_{j=1}^{m} f\left(z_{j}\right)\right\}^{1 / 2}-\frac{r^{2}}{4}\left\{\prod_{j=1}^{m} f\left(z_{j}\right)\right\}^{1 / 2}\right. \\
\left.\times\left\{\sum_{j=1}^{m} \psi_{f}\left(z_{j}\right) x_{j}^{2}+\sum_{j=1}^{m} \sum_{\substack{l=1 \\
l \neq j}}^{m} \phi_{f}\left(z_{j}\right) \phi_{f}\left(z_{l}\right) x_{j} x_{l}\right\}\right]^{2} d \mathbf{z} .
\end{aligned}
$$

In order to prove (i), it is thus sufficient to establish differentiability in quadratic mean with respect to $r^{2}$. This quadratic mean differentiability property, however, is somewhat nonstandard, as it involves the second-order derivatives of the product $\left(\prod_{j=1}^{m} f\left(z_{j}\right)\right)$. As in Akharif and Hallin (2003), the proof is decomposed into three parts.

(a) $y^{2} \mapsto l_{z, x}(y):=\int_{\mathbb{R}} G_{z, x}(\eta, y) h(\eta) d \eta$, with $G_{z, x}(\eta, y)=\prod_{j=1}^{m} f\left(z_{j}-x_{j} y \eta\right)$ is absolutely continuous in a right-neighborhood of $y=0$ with a.e. derivative

$$
\begin{aligned}
D_{z}(y) & =\frac{1}{2 y} \int_{w=0}^{y} \int_{\mathbb{R}}\left[\sum_{j=1}^{m} \ddot{f}\left(z_{j}-x_{j} w \eta\right) x_{j}^{2} \prod_{\substack{l=1 \\
l \neq j}}^{m} f\left(z_{l}-x_{l} w \eta\right)\right. \\
& \left.+\sum_{j=1}^{m} \sum_{\substack{l=1 \\
l \neq j}}^{m} \dot{f}\left(z_{j}-x_{j} w \eta\right) \dot{f}\left(z_{l}-x_{l} w \eta\right) x_{j} x_{l} \prod_{\substack{s=1 \\
s \neq l \\
s \neq j}}^{m} f\left(z_{s}-x_{s} w \eta\right)\right] \eta^{2} h(\eta) d \eta d w .
\end{aligned}
$$


We obtain

$$
\begin{aligned}
l_{z, x}(y)-l_{z, x}(0)= & \int_{\mathbb{R}}\left(G_{z, x}(\eta, y)-G_{z, x}(\eta, 0)\right) h(\eta) d \eta \\
= & \int_{\mathbb{R}} \int_{b=0}^{y} \dot{G}_{z, x}(\eta, b) d b h(\eta) d \eta \\
= & \int_{\mathbb{R}} \int_{b=0}^{y}\left(\dot{G}_{z, x}(\eta, b)-\dot{G}_{z, x}(\eta, 0)\right) d b h(\eta) d \eta \\
& +\int_{\mathbb{R}} \int_{b=0}^{y} \dot{G}_{z, x}(\eta, 0) d b h(\eta) d \eta \\
= & \int_{\mathbb{R}} \int_{b=0}^{y} \int_{w=0}^{b} \ddot{G}_{z, x}(\eta, w) d w d b h(\eta) d \eta \\
= & \frac{1}{2} \int_{a=0}^{y^{2}} a^{\frac{-1}{2}} \int_{w=0}^{a^{\frac{1}{2}}} \int_{\mathbb{R}} \ddot{G}_{z, x}(\eta, w) h(\eta) d \eta d w d a,
\end{aligned}
$$

where

$$
\begin{aligned}
\ddot{G}_{z, x}(\eta, w):= & \sum_{j=1}^{m} \ddot{f}\left(z_{j}-x_{j} w \eta\right) x_{j}^{2} \eta^{2} \prod_{\substack{l=1 \\
l \neq j}}^{m} f\left(z_{l}-x_{l} w \eta\right) \\
& +\sum_{j=1}^{m} \sum_{\substack{l=1 \\
l \neq j}}^{m} \dot{f}\left(z_{j}-x_{j} w \eta\right) \dot{f}\left(z_{l}-x_{l} w \eta\right) x_{j} x_{l} \eta^{2} \prod_{\substack{s=1 \\
s \neq l \\
s \neq j}}^{m} f\left(z_{s}-x_{s} w \eta\right) .
\end{aligned}
$$

The value (A.2) of the a.e. derivative for $y>0$ follows. At $y=0$, the right derivative is defined as the limit, as $y \longrightarrow 0$, of $\left(l_{z, x}(y)-l_{z, x}(0)\right) / y^{2}$, for which (A.3) yields 0/0. Applying L'Hospital's rule,

$$
\frac{1}{2}\left[\sum_{j=1}^{m} \ddot{f}\left(z_{j}\right) x_{j}^{2} \prod_{\substack{l=1 \\ l \neq j}}^{m} f\left(z_{l}\right)+\sum_{j=1}^{m} \sum_{\substack{l=1 \\ l \neq j}}^{m} \dot{f}\left(z_{j}\right) \dot{f}\left(z_{l}\right) x_{j} x_{l} \prod_{\substack{s=1 \\ s \neq l \\ s \neq j}}^{m} f\left(z_{s}\right)\right]
$$

(b) It follows that $y^{2} \mapsto s_{z, x}(y):=\left[l_{z, x}(y)\right]^{1 / 2}$ is absolutely continuous in a neighborhood of $y=0$, with a.e. derivative

$$
\dot{s}_{z, x}(y)=\frac{1}{4 y} \int_{w=0}^{y} \frac{\int_{\mathbb{R}} \ddot{G}_{z, x}(\eta, w) h(\eta) d \eta}{\left[\int_{\mathbb{R}} G_{z}(\eta, y) h(\eta) d \eta\right]^{\frac{1}{2}}} d w .
$$

L'Hospital's rule at $y=0$ yields

$\dot{s}_{z, x}(0)=\frac{1}{4}\left(\prod_{j=1}^{m} f\left(z_{j}\right)\right)^{\frac{-1}{2}}\left\{\sum_{j=1}^{m} \ddot{f}\left(z_{j}\right) x_{j}^{2} \prod_{\substack{l=1 \\ l \neq j}}^{m} f\left(z_{l}\right)+\sum_{j=1}^{m} \sum_{\substack{l=1 \\ l \neq j}}^{m} \dot{f}\left(z_{j}\right) \dot{f}\left(z_{l}\right) x_{j} x_{\substack { s \\ \begin{subarray}{c}{s=1 \\ s \neq l \\ s \neq j{ s \\ \begin{subarray} { c } { s = 1 \\ s \neq l \\ s \neq j } }\end{subarray}}^{m} f\left(z_{s}\right)\right\}$.

Consequently, for all $z$

$$
\lim _{y \rightarrow 0}\left[s_{z, x}(y)-s_{z, x}(0)\right] / y^{2}=\dot{s}_{z, x}(0) .
$$

(c) The partial quadratic mean differentiablity property to be proved takes the form :

$$
\lim _{y \rightarrow 0} \int_{\mathbb{R}^{m}}\left\{\frac{1}{y^{2}}\left[s_{z, x}(y)-s_{z, x}(0)\right]-\dot{s}_{z, x}(0)\right\}^{2} d \mathbf{z}=0 .
$$

From (b) abov,

$$
\left\{\frac{1}{y^{2}}\left[s_{z, x}(y)-s_{z, x}(0)\right]\right\}^{2}=\left(\frac{1}{y^{2}}\right)^{2}\left(\int_{\lambda=0}^{y^{2}} \dot{s}_{z, x}(\sqrt{\lambda}) d \lambda\right)^{2}
$$


for all z. Fubini's theorem and (A.4) yield

$$
\begin{aligned}
\int_{\mathbb{R}^{m}}\left\{\frac{1}{y^{2}}\left[s_{z, x}(y)-s_{z, x}(0)\right]\right\}^{2} d \mathbf{z} & \leq \frac{1}{y^{2}} \int_{\lambda=0}^{y^{2}} \int_{\mathbb{R}^{m}}\left(\dot{s}_{z, x}(\sqrt{\lambda})\right)^{2} d \mathbf{z} d \lambda \\
& =\frac{1}{16 y^{2}} \int_{\lambda=0}^{y^{2}} \mathcal{I}_{\psi \phi}^{x}(f ; \sqrt{\lambda}) d \lambda
\end{aligned}
$$

with $\mathcal{I}_{\psi \phi}^{x}$ defined (2.1). From the continuity assumption in (C.2), this latter quantity converges, as $y \rightarrow 0$, to $\mathcal{I}_{\psi \phi}^{x}(f ; 0) / 16=\int_{\mathbb{R}^{m}}\left(\dot{s}_{z, x}(0)\right)^{2} d \mathbf{z}$, which together with (A.7), entails that

$$
\limsup _{y \rightarrow 0} \int_{\mathbb{R}^{m}}\left\{\frac{1}{y^{2}}\left[s_{z, x}(y)-s_{z, x}(0)\right]\right\}^{2} d \mathbf{z} \leq \int_{\mathbb{R}^{m}}\left(\dot{s}_{z, x}(0)\right)^{2} d \mathbf{z}
$$

In view of Theorem V.I.3 of Hájek and Šidák (1967), (A.5) and (A.8) jointly imply (A.6). This completes the proof of (i).

(ii) The problem here reduces to the classical case of linear models considered by [26].

(iii) First note that, as $t, s \rightarrow 0$,

$$
\int_{\mathbb{R}^{m}}\left\{D_{\sigma_{\eta}^{2}} q_{\beta_{0}+t, \beta_{1}+s, \sigma^{2}+v, \sigma_{\eta}^{2} ; f_{1}}^{1 / 2}(y)_{\mid \sigma_{\eta}^{2}=0}-D_{\sigma_{\eta}^{2}} q_{\beta_{0}, \beta_{1}, \sigma^{2}, \sigma_{\eta}^{2} ; f_{1}}^{1 / 2}(y)_{\mid \sigma_{\eta}^{2}=0}\right\}^{2} d y=o(1) .
$$

For the perturbation of $\sigma^{2}$, let $z_{j}=y_{j}-\beta_{0}-\beta_{1} x_{j}$ for $j=1,2, \ldots, m$, we have

$$
\begin{aligned}
& Q_{\sigma^{2}}:=\int_{\mathbb{R}^{m}}\left\{D_{\sigma_{\eta}^{2}} q_{\beta_{0}+t, \beta_{1}+s, \sigma^{2}+v, \sigma_{\eta}^{2} ; f_{1}}^{1 / 2}(y)_{\mid \sigma_{\eta}^{2}=0}-D_{\sigma_{\eta}^{2}} q_{\beta_{0}, \beta_{1}, \sigma^{2}, \sigma_{\eta}^{2} ; f_{1}}^{1 / 2}(y)_{\mid \sigma_{\eta}^{2}=0}\right\}^{2} d y \\
& =\int_{\mathbb{R}^{m}}\left\{\frac{1}{4\left(\sigma^{2}+v\right)^{(m+4) / 4}}\left[\prod_{j=1}^{m} f_{1}^{1 / 2}\left(\frac{y_{j}-\beta_{0}-\beta_{1} x_{j}}{\left(\sigma^{2}+v\right)^{1 / 2}}\right)\right]\right. \\
& \times\left[\sum_{j=1}^{m} \psi_{f_{1}}\left(\frac{y_{j}-\beta_{0}-\beta_{1} x_{j}}{\left(\sigma^{2}+v\right)^{1 / 2}}\right) x_{j}^{2}+\sum_{j=1}^{m} \sum_{\substack{l=1 \\
l \neq j}}^{m} \phi_{f_{1}}\left(\frac{y_{j}-\beta_{0}-\beta_{1} x_{j}}{\left(\sigma^{2}+v\right)^{1 / 2}}\right) \phi_{f_{1}}\left(\frac{y_{l}-\beta_{0}-\beta_{1} x_{l}}{\left(\sigma^{2}+v\right)^{1 / 2}}\right) x_{j} x_{l}\right] \\
& -\frac{1}{4\left(\sigma^{2}\right)^{(m+4) / 4}}\left[\prod_{j=1}^{m} f_{1}^{1 / 2}\left(\frac{y_{j}-\beta_{0}-\beta_{1} x_{j}}{\left(\sigma^{2}\right)^{1 / 2}}\right)\right] \\
& \left.\times\left[\sum_{j=1}^{m} \psi_{f_{1}}\left(\frac{y_{j}-\beta_{0}-\beta_{1} x_{j}}{\left(\sigma^{2}\right)^{1 / 2}}\right) x_{j}^{2}+\sum_{j=1}^{m} \sum_{\substack{l=1 \\
l \neq j}}^{m} \phi_{f_{1}}\left(\frac{y_{j}-\beta_{0}-\beta_{1} x_{j}}{\left(\sigma^{2}\right)^{1 / 2}}\right) \phi_{f_{1}}\left(\frac{y_{l}-\beta_{0}-\beta_{1} x_{l}}{\left(\sigma^{2}\right)^{1 / 2}}\right) x_{j} x_{l}\right]\right\}^{2} d y \\
& =\int_{\mathbb{R}^{m}}\left\{\frac{1}{4\left(\sigma^{2}+v\right)^{(m+4) / 4}}\left[\prod_{j=1}^{m} f_{1}^{1 / 2}\left(\frac{z_{j}}{\left(\sigma^{2}+v\right)^{1 / 2}}\right)\right]\right. \\
& \times\left[\sum_{j=1}^{m} \psi_{f_{1}}\left(\frac{z_{j}}{\left(\sigma^{2}+v\right)^{1 / 2}}\right) x_{j}^{2}+\sum_{j=1}^{m} \sum_{\substack{l=1 \\
l \neq j}}^{m} \phi_{f_{1}}\left(\frac{z_{j}}{\left(\sigma^{2}+v\right)^{1 / 2}}\right) \phi_{f_{1}}\left(\frac{z_{l}}{\left(\sigma^{2}+v\right)^{1 / 2}}\right) x_{j} x_{l}\right] \\
& -\frac{1}{4\left(\sigma^{2}\right)^{(m+4) / 4}}\left[\prod_{j=1}^{m} f_{1}^{1 / 2}\left(\frac{z_{j}}{\left(\sigma^{2}\right)^{1 / 2}}\right)\right] \\
& \left.\times\left[\sum_{j=1}^{m} \psi_{f_{1}}\left(\frac{z_{j}}{\left(\sigma^{2}\right)^{1 / 2}}\right) x_{j}^{2}+\sum_{j=1}^{m} \sum_{\substack{l=1 \\
l \neq j}}^{m} \phi_{f_{1}}\left(\frac{z_{j}}{\left(\sigma^{2}\right)^{1 / 2}}\right) \phi_{f_{1}}\left(\frac{z_{l}}{\left(\sigma^{2}\right)^{1 / 2}}\right) x_{j} x_{l}\right]\right\}^{2} d \mathbf{z}
\end{aligned}
$$




$$
\begin{aligned}
& =\int_{\mathbb{R}^{m}}\left\{\frac{1}{4}\left[\frac{1}{\left(\sigma^{2}+v\right)} \prod_{j=1}^{m} \frac{1}{\left(\sigma^{2}+v\right)^{1 / 4}} f_{1}^{1 / 2}\left(\frac{z_{j}}{\left(\sigma^{2}+v\right)^{1 / 2}}\right)-\frac{1}{\sigma^{2}} \prod_{j=1}^{m} \frac{1}{\sigma^{1 / 2}} f_{1}^{1 / 2}\left(\frac{z_{j}}{\sigma}\right)\right]\right. \\
& \times\left[\sum_{j=1}^{m} \psi_{f_{1}}\left(\frac{z_{j}}{\left(\sigma^{2}+v\right)^{1 / 2}}\right) x_{j}^{2}+\sum_{j=1}^{m} \sum_{\substack{l=1 \\
l \neq j}}^{m} \phi_{f_{1}}\left(\frac{z_{j}}{\left(\sigma^{2}+v\right)^{1 / 2}}\right) \phi_{f_{1}}\left(\frac{z_{l}}{\left(\sigma^{2}+v\right)^{1 / 2}}\right) x_{j} x_{l}\right] \\
& +\frac{1}{4} \frac{1}{\sigma^{2}}\left[\prod_{j=1}^{m} \frac{1}{\left(\sigma^{2}\right)^{1 / 4}} f_{1}^{1 / 2}\left(\frac{z_{j}}{\sigma}\right)\right]\left\{\sum_{j=1}^{m}\left[\psi_{f_{1}}\left(\frac{z_{j}}{\left(\sigma^{2}+v\right)^{1 / 2}}\right)-\psi_{f_{1}}\left(\frac{z_{j}}{\sigma}\right)\right] x_{j}^{2}\right. \\
& \left.\left.+\sum_{j=1}^{m} \sum_{\substack{l=1 \\
l \neq j}}^{m}\left[\phi_{f_{1}}\left(\frac{z_{j}}{\left(\sigma^{2}+v\right)^{1 / 2}}\right) \phi_{f_{1}}\left(\frac{z_{l}}{\left(\sigma^{2}+v\right)^{1 / 2}}\right)-\phi_{f_{1}}\left(\frac{z_{j}}{\sigma}\right) \phi_{f_{1}}\left(\frac{z_{l}}{\sigma}\right)\right] x_{j} x_{l}\right\}\right\}^{2} d \mathbf{z} \\
& =\int_{\mathbb{R}^{m}}\left\{\frac{1}{4}\left[\frac{1}{\left(\sigma^{2}+v\right)}-\frac{1}{\sigma^{2}}\right] \prod_{j=1}^{m} \frac{1}{\left(\sigma^{2}+v\right)^{1 / 4}} f_{1}^{1 / 2}\left(\frac{z_{j}}{\left(\sigma^{2}+v\right)^{1 / 2}}\right)\right. \\
& \times\left[\sum_{j=1}^{m} \psi_{f_{1}}\left(\frac{z_{j}}{\left(\sigma^{2}+v\right)^{1 / 2}}\right) x_{j}^{2}+\sum_{j=1}^{m} \sum_{\substack{l=1 \\
l \neq j}}^{m} \phi_{f_{1}}\left(\frac{z_{j}}{\left(\sigma^{2}+v\right)^{1 / 2}}\right) \phi_{f_{1}}\left(\frac{z_{l}}{\left(\sigma^{2}+v\right)^{1 / 2}}\right) x_{j} x_{l}\right] \\
& +\frac{1}{4}\left[\frac{1}{\sigma^{2}} \prod_{j=1}^{m} \frac{1}{\left(\sigma^{2}+v\right)^{1 / 4}} f_{1}^{1 / 2}\left(\frac{z_{j}}{\left(\sigma^{2}+v\right)^{1 / 2}}\right)-\frac{1}{\sigma^{2}} \prod_{j=1}^{m} \frac{1}{\sigma^{1 / 2}} f_{1}^{1 / 2}\left(\frac{z_{j}}{\sigma}\right)\right] \\
& \times\left[\sum_{j=1}^{m} \psi_{f_{1}}\left(\frac{z_{j}}{\left(\sigma^{2}+v\right)^{1 / 2}}\right) x_{j}^{2}+\sum_{j=1}^{m} \sum_{\substack{l=1 \\
l \neq j}}^{m} \phi_{f_{1}}\left(\frac{z_{j}}{\left(\sigma^{2}+v\right)^{1 / 2}}\right) \phi_{f_{1}}\left(\frac{z_{l}}{\left(\sigma^{2}+v\right)^{1 / 2}}\right) x_{j} x_{l}\right] \\
& +\frac{1}{4} \frac{1}{\sigma^{2}}\left[\prod_{j=1}^{m} \frac{1}{\left(\sigma^{2}\right)^{1 / 4}} f_{1}^{1 / 2}\left(\frac{z_{j}}{\sigma}\right)\right]\left\{\sum_{j=1}^{m}\left[\psi_{f_{1}}\left(\frac{z_{j}}{\left(\sigma^{2}+v\right)^{1 / 2}}\right)-\psi_{f_{1}}\left(\frac{z_{j}}{\sigma}\right)\right] x_{j}^{2}\right. \\
& \left.\left.+\sum_{j=1}^{m} \sum_{\substack{l=1 \\
l \neq j}}^{m}\left[\phi_{f_{1}}\left(\frac{z_{j}}{\left(\sigma^{2}+v\right)^{1 / 2}}\right) \phi_{f_{1}}\left(\frac{z_{l}}{\left(\sigma^{2}+v\right)^{1 / 2}}\right)-\phi_{f_{1}}\left(\frac{z_{j}}{\sigma}\right) \phi_{f_{1}}\left(\frac{z_{l}}{\sigma}\right)\right] x_{j} x_{l}\right\}\right\}^{2} d \mathbf{z} \\
& \leq C\left(Q_{1}+Q_{2}+Q_{3}\right) .
\end{aligned}
$$

where

$$
\begin{aligned}
& Q_{1}:=\int_{\mathbb{R}^{m}}\left\{\frac{1}{4}\left[\frac{1}{\left(\sigma^{2}+v\right)}-\frac{1}{\sigma^{2}}\right] \prod_{j=1}^{m} \frac{1}{\left(\sigma^{2}+v\right)^{1 / 4}} f_{1}^{1 / 2}\left(\frac{z_{j}}{\left(\sigma^{2}+v\right)^{1 / 2}}\right)\right. \\
& \left.\times\left[\sum_{j=1}^{m} \psi_{f_{1}}\left(\frac{z_{j}}{\left(\sigma^{2}+v\right)^{1 / 2}}\right) x_{j}^{2}+\sum_{j=1}^{m} \sum_{\substack{l=1 \\
l \neq j}}^{m} \phi_{f_{1}}\left(\frac{z_{j}}{\left(\sigma^{2}+v\right)^{1 / 2}}\right) \phi_{f_{1}}\left(\frac{z_{l}}{\left(\sigma^{2}+v\right)^{1 / 2}}\right) x_{j} x_{l}\right]\right\}^{2} d \mathbf{z}, \\
& Q_{2}:=\int_{\mathbb{R}^{m}}\left\{\frac{1}{4 \sigma^{2}}\left[\prod_{j=1}^{m} \frac{1}{\left(\sigma^{2}+v\right)^{1 / 4}} f_{1}^{1 / 2}\left(\frac{z_{j}}{\left(\sigma^{2}+v\right)^{1 / 2}}\right)-\frac{1}{\sigma^{2}} \prod_{j=1}^{m} \frac{1}{\sigma^{1 / 2}} f_{1}^{1 / 2}\left(\frac{z_{j}}{\sigma}\right)\right]\right. \\
& \left.\times\left[\sum_{j=1}^{m} \psi_{f_{1}}\left(\frac{z_{j}}{\left(\sigma^{2}+v\right)^{1 / 2}}\right) x_{j}^{2}+\sum_{j=1}^{m} \sum_{\substack{l=1 \\
l \neq j}}^{m} \phi_{f_{1}}\left(\frac{z_{j}}{\left(\sigma^{2}+v\right)^{1 / 2}}\right) \phi_{f_{1}}\left(\frac{z_{l}}{\left(\sigma^{2}+v\right)^{1 / 2}}\right) x_{j} x_{l}\right]\right\}^{2} d \mathbf{z},
\end{aligned}
$$

and

$$
\begin{aligned}
Q_{3} & :=\int_{\mathbb{R}^{m}}\left\{\frac { 1 } { 4 \sigma ^ { 2 } } [ \prod _ { j = 1 } ^ { m } \frac { 1 } { ( \sigma ^ { 2 } ) ^ { 1 / 4 } } f _ { 1 } ^ { 1 / 2 } ( \frac { z _ { j } } { \sigma } ) ] \left\{\sum_{j=1}^{m}\left[\psi_{f_{1}}\left(\frac{z_{j}}{\left(\sigma^{2}+v\right)^{1 / 2}}\right)-\psi_{f_{1}}\left(\frac{z_{j}}{\sigma}\right)\right] x_{j}^{2}\right.\right. \\
+ & \left.\left.\sum_{j=1}^{m} \sum_{\substack{l=1 \\
l \neq j}}^{m}\left[\phi_{f_{1}}\left(\frac{z_{j}}{\left(\sigma^{2}+v\right)^{1 / 2}}\right) \phi_{f_{1}}\left(\frac{z_{l}}{\left(\sigma^{2}+v\right)^{1 / 2}}\right)-\phi_{f_{1}}\left(\frac{z_{j}}{\sigma}\right) \phi_{f_{1}}\left(\frac{z_{l}}{\sigma}\right)\right] x_{j} x_{l}\right\}\right\}^{2} d \mathbf{z} .
\end{aligned}
$$


Clearly, $Q_{1}=O\left(\left[\left(\sigma^{2}+v\right)^{-1}-\sigma^{-2}\right]^{2}\left(\mathcal{I}_{\psi}\left(f_{1}\right) \overline{x^{2}}+\mathcal{I}_{\phi}^{2}\left(f_{1}\right)\left(m(\bar{x})^{2}-\overline{x^{2}}\right)\right)\right)$, which implies that $Q_{1}=o(1)$, as $v \longrightarrow 0$.

With regard to $Q_{2}$, we have

$$
\begin{aligned}
Q_{2}= & \int_{\mathbb{R}^{m}}\left\{\frac { 1 } { 4 \sigma ^ { 2 } } \left\{\sum_{j=1}^{m}\left[\frac{1}{\left(\sigma^{2}+v\right)^{1 / 4}} f_{1}^{1 / 2}\left(\frac{z_{j}}{\left(\sigma^{2}+v\right)^{1 / 2}}\right)-\frac{1}{\sigma^{1 / 2}} f_{1}^{1 / 2}\left(\frac{z_{j}}{\sigma}\right)\right]\right.\right. \\
& \times \prod_{k=1}^{j-1} \frac{1}{\left(\sigma^{2}+v\right)^{1 / 4}} f_{1}^{1 / 2}\left(\frac{z_{k}}{\left(\sigma^{2}+v\right)^{1 / 2}}\right) \prod_{k=j+1}^{m} \frac{1}{\left(\sigma^{2}\right)^{1 / 4}} f_{1}^{1 / 2}\left(\frac{z_{k}}{\sigma}\right) \\
& \left.\left.\times\left[\sum_{j=1}^{m} \psi_{f_{1}}\left(\frac{z_{j}}{\left(\sigma^{2}+v\right)^{1 / 2}}\right) x_{j}^{2}+\sum_{j=1}^{m} \sum_{\substack{l=1 \\
l \neq j}}^{m} \phi_{f_{1}}\left(\frac{z_{j}}{\left(\sigma^{2}+v\right)^{1 / 2}}\right) \phi_{f_{1}}\left(\frac{z_{l}}{\left(\sigma^{2}+v\right)^{1 / 2}}\right) x_{j} x_{l}\right]\right\}\right\}^{2} d \mathbf{z} \\
= & \int_{\mathbb{R}^{m}}\left\{\frac { 1 } { 4 \sigma ^ { 2 } } \left\{\sum_{j=1}^{m} \sum_{l=1}^{m}\left[\frac{1}{\left(\sigma^{2}+v\right)^{1 / 4}} f_{1}^{1 / 2}\left(\frac{z_{j}}{\left(\sigma^{2}+v\right)^{1 / 2}}\right)-\frac{1}{\sigma^{1 / 2}} f_{1}^{1 / 2}\left(\frac{z_{j}}{\sigma}\right)\right]\right.\right. \\
& \left.\times \psi_{f_{1}}\left(\frac{z_{l}}{\left(\sigma^{2}+v\right)^{1 / 2}}\right) x_{l}^{2} \prod_{k=1}^{j-1} \frac{1}{\left(\sigma^{2}+v\right)^{1 / 4}} f_{1}^{1 / 2}\left(\frac{z_{k}}{\left(\sigma^{2}+v\right)^{1 / 2}}\right) \prod_{k=j+1}^{m} \frac{1}{\left(\sigma^{2}\right)^{1 / 4}} f_{1}^{1 / 2}\left(\frac{z_{k}}{\sigma}\right)\right\} \\
& +\frac{1}{4 \sigma^{2}} \sum_{j=1}^{m} \sum_{l=1}^{m} \sum_{\substack{t=1 \\
t \neq l}}^{m}\left[\frac{1}{\left(\sigma^{2}+v\right)^{1 / 4}} f_{1}^{1 / 2}\left(\frac{z_{j}}{\left(\sigma^{2}+v\right)^{1 / 2}}\right)-\frac{1}{\sigma^{1 / 2}} f_{1}^{1 / 2}\left(\frac{z_{j}}{\sigma}\right)\right] \phi_{f_{1}}\left(\frac{z_{l}}{\left(\sigma^{2}+v\right)^{1 / 2}}\right) \\
& \left.\times \phi_{f_{1}}\left(\frac{z_{t}}{\left(\sigma^{2}+v\right)^{1 / 2}}\right) x_{l} x_{t} \prod_{k=1}^{j-1} \frac{1}{\left(\sigma^{2}+v\right)^{1 / 4}} f_{1}^{1 / 2}\left(\frac{z_{k}}{\left(\sigma^{2}+v\right)^{1 / 2}}\right) \prod_{k=j+1}^{m} \frac{1}{\left(\sigma^{2}\right)^{1 / 4}} f_{1}^{1 / 2}\left(\frac{z_{k}}{\sigma}\right)\right\}^{2} d \mathbf{z} \\
\leq & C_{1}\left(Q_{2}^{1}+Q_{2}^{2}\right)
\end{aligned}
$$

where

$$
\begin{aligned}
Q_{2}^{1}:= & \int_{\mathbb{R}^{m}}\left\{\frac { 1 } { 4 \sigma ^ { 2 } } \left\{\sum_{j=1}^{m} \sum_{l=1}^{m}\left[\frac{1}{\left(\sigma^{2}+v\right)^{1 / 4}} f_{1}^{1 / 2}\left(\frac{z_{j}}{\left(\sigma^{2}+v\right)^{1 / 2}}\right)-\frac{1}{\sigma^{1 / 2}} f_{1}^{1 / 2}\left(\frac{z_{j}}{\sigma}\right)\right]\right.\right. \\
& \left.\left.\times \psi_{f_{1}}\left(\frac{z_{l}}{\left(\sigma^{2}+v\right)^{1 / 2}}\right) x_{l}^{2} \prod_{k=1}^{j-1} \frac{1}{\left(\sigma^{2}+v\right)^{1 / 4}} f_{1}^{1 / 2}\left(\frac{z_{k}}{\left(\sigma^{2}+v\right)^{1 / 2}}\right) \prod_{k=j+1}^{m} \frac{1}{\left(\sigma^{2}\right)^{1 / 4}} f_{1}^{1 / 2}\left(\frac{z_{k}}{\sigma}\right)\right\}\right\}^{2} d \mathbf{z}
\end{aligned}
$$

and

$$
\begin{aligned}
Q_{2}^{2} & :=\int_{\mathbb{R}^{m}}\left\{\frac{1}{4 \sigma^{2}} \sum_{j=1}^{m} \sum_{l=1}^{m} \sum_{\substack{t=1 \\
t \neq l}}^{m}\left[\frac{1}{\left(\sigma^{2}+v\right)^{1 / 4}} f_{1}^{1 / 2}\left(\frac{z_{j}}{\left(\sigma^{2}+v\right)^{1 / 2}}\right)-\frac{1}{\sigma^{1 / 2}} f_{1}^{1 / 2}\left(\frac{z_{j}}{\sigma}\right)\right] \phi_{f_{1}}\left(\frac{z_{l}}{\left(\sigma^{2}+v\right)^{1 / 2}}\right)\right. \\
& \left.\times \phi_{f_{1}}\left(\frac{z_{t}}{\left(\sigma^{2}+v\right)^{1 / 2}}\right) x_{l} x_{t} \prod_{k=1}^{j-1} \frac{1}{\left(\sigma^{2}+v\right)^{1 / 4}} f_{1}^{1 / 2}\left(\frac{z_{k}}{\left(\sigma^{2}+v\right)^{1 / 2}}\right) \prod_{k=j+1}^{m} \frac{1}{\left(\sigma^{2}\right)^{1 / 4}} f_{1}^{1 / 2}\left(\frac{z_{k}}{\sigma}\right)\right\}^{2} d \mathbf{z} .
\end{aligned}
$$

To show that $Q_{2}=o(1)$ as $v \longrightarrow 0$, it is clearly sufficient to prove that $Q_{2}^{2}$ and $Q_{2}^{1}$ are $o(1)$. We begin with $Q_{2}^{1}$, which is bounded by $A\left(Q_{2}^{11}+Q_{2}^{12}+Q_{2}^{13}\right)$, where $A$ is some positive constant. 


$$
\begin{aligned}
& Q_{2}^{11}:=\int_{\mathbb{R}^{m}}\left\{\sum _ { j = 1 } ^ { m } \left[\frac{1}{\left(\sigma^{2}+v\right)^{1 / 4}} f_{1}^{1 / 2}\left(\frac{z_{j}}{\left(\sigma^{2}+v\right)^{1 / 2}}\right) \psi_{f_{1}}\left(\frac{z_{j}}{\left(\sigma^{2}+v\right)^{1 / 2}}\right) x_{j}^{2}-\right.\right. \\
& \left.\frac{1}{\sigma^{1 / 2}} f_{1}^{1 / 2}\left(\frac{z_{j}}{\sigma}\right) \psi_{f_{1}}\left(\frac{z_{j}}{\sigma}\right) x_{j}^{2}\right] \prod_{k=1}^{j-1} \frac{1}{\left(\sigma^{2}+v\right)^{1 / 4}} f_{1}^{1 / 2}\left(\frac{z_{k}}{\left(\sigma^{2}+v\right)^{1 / 2}}\right) \\
& \left.\times \prod_{k=j+1}^{m} \frac{1}{\left(\sigma^{2}\right)^{1 / 4}} f_{1}^{1 / 2}\left(\frac{z_{k}}{\sigma}\right)\right\}^{2} d \mathbf{z} \\
& \leq A_{1} \int_{\mathbb{R}}\left\{e^{\frac{1}{2}\left[u-\ln \left(1+\frac{v}{\sigma^{2}}\right)^{1 / 2}\right]} f_{1}^{1 / 2}\left(e^{u-\ln \left(1+\frac{v}{\sigma^{2}}\right)^{1 / 2}}\right) \psi_{f_{1}}\left(e^{u-\ln \left(1+\frac{v}{\sigma^{2}}\right)^{1 / 2}}\right)\right. \\
& \left.-e^{\frac{1}{2} u} f_{1}^{1 / 2}\left(e^{u}\right) \psi_{f_{1}}\left(e^{u}\right)\right\}^{2} d u \\
& Q_{2}^{12}:=\int_{\mathbb{R}^{m}}\left\{\sum_{j=1}^{m}\left[\psi_{f_{1}}\left(\frac{z_{j}}{\left(\sigma^{2}+v\right)^{1 / 2}}\right)-\psi_{f_{1}}\left(\frac{z_{j}}{\sigma}\right)\right] x_{j}^{2} \frac{1}{\sigma^{1 / 2}} f_{1}^{1 / 2}\left(\frac{z_{j}}{\sigma}\right)\right. \\
& \left.\times \prod_{k=1}^{j-1} \frac{1}{\left(\sigma^{2}+v\right)^{1 / 4}} f_{1}^{1 / 2}\left(\frac{z_{k}}{\left(\sigma^{2}+v\right)^{1 / 2}}\right) \prod_{k=j+1}^{m} \frac{1}{\left(\sigma^{2}\right)^{1 / 4}} f_{1}^{1 / 2}\left(\frac{z_{k}}{\sigma}\right)\right\}^{2} d \mathbf{z} \\
& \leq A_{2} \int_{\mathbb{R}}\left\{\psi_{f_{1}}\left(e^{u-\ln \left(1+\frac{v}{\sigma^{2}}\right)^{1 / 2}}\right)-\psi_{f_{1}}\left(e^{u}\right)\right\}^{2} e^{u} f_{1}\left(e^{u}\right) d u \\
& Q_{2}^{13}:=\int_{\mathbb{R}^{m}}\left\{2 \sum_{j=2}^{m} \sum_{l=1}^{m-1}\left[\frac{1}{\left(\sigma^{2}+v\right)^{1 / 4}} f_{1}^{1 / 2}\left(\frac{z_{j}}{\left(\sigma^{2}+v\right)^{1 / 2}}\right)-\frac{1}{\sigma^{1 / 2}} f_{1}^{1 / 2}\left(\frac{z_{j}}{\sigma}\right)\right]\right. \\
& \left.\psi_{f_{1}}\left(\frac{z_{l}}{\left(\sigma^{2}+v\right)^{1 / 2}}\right) x_{l}^{2} \times \prod_{k=1}^{j-1} \frac{1}{\left(\sigma^{2}+v\right)^{1 / 4}} f_{1}^{1 / 2}\left(\frac{z_{k}}{\left(\sigma^{2}+v\right)^{1 / 2}}\right) \prod_{k=j+1}^{m} \frac{1}{\left(\sigma^{2}\right)^{1 / 4}} f_{1}^{1 / 2}\left(\frac{z_{k}}{\sigma}\right)\right\}^{2} d \mathbf{z} \\
& \leq A_{3} \int_{\mathbb{R}}\left\{e^{\frac{1}{2}\left[u-\ln \left(1+\frac{v}{\sigma^{2}}\right)^{1 / 2}\right]} f_{1}^{1 / 2}\left(e^{u-\ln \left(1+\frac{v}{\sigma^{2}}\right)^{1 / 2}}\right)-e^{\frac{1}{2} u} f_{1}^{1 / 2}\left(e^{u}\right)\right\}^{2} d u \times \mathcal{I}_{\psi}\left(f_{1}\right) \overline{x^{2}} .
\end{aligned}
$$

As $e^{\frac{1}{2} u} f_{1}^{1 / 2}\left(e^{u}\right), e^{\frac{1}{2} u} f_{1}^{1 / 2}\left(e^{u}\right) \psi_{f_{1}}\left(e^{u}\right)$ and $\psi_{f_{1}}\left(e^{u}\right)$ are square integrable, quadratic mean continuity implies that the integrals in (A.10), (A.11) and (A.12) are $o(1)$ as $h \longrightarrow 0$. In the same way, it is easily shown that:

$$
\begin{aligned}
Q_{2}^{11} \leq & B\left[\int_{\mathbb{R}}\left\{e^{\frac{1}{2}\left[u-\ln \left(1+\frac{v}{\sigma^{2}}\right)^{1 / 2}\right]} f_{1}^{1 / 2}\left(e^{u-\ln \left(1+\frac{v}{\sigma^{2}}\right)^{1 / 2}}\right) \phi_{f_{1}}\left(e^{u-\ln \left(1+\frac{v}{\sigma^{2}}\right)^{1 / 2}}\right)-e^{\frac{1}{2} u} f_{1}^{1 / 2}\left(e^{u}\right) \phi_{f_{1}}\left(e^{u}\right)\right\}^{2} d u\right. \\
& \times \mathcal{I}_{\phi}\left(f_{1}\right) \bar{x}+\int_{\mathbb{R}}\left\{\phi_{f_{1}}\left(e^{u-\ln \left(1+\frac{v}{\sigma^{2}}\right)^{1 / 2}}\right)-\phi_{f_{1}}\left(e^{u}\right)\right\}^{2} e^{u} f_{1}\left(e^{u}\right) d u \times \mathcal{I}_{\phi}\left(f_{1}\right) \bar{x} \\
& \left.+\int_{\mathbb{R}}\left\{e^{\frac{1}{2}\left[u-\ln \left(1+\frac{v}{\sigma^{2}}\right)^{1 / 2}\right]} f_{1}^{1 / 2}\left(e^{u-\ln \left(1+\frac{v}{\sigma^{2}}\right)^{1 / 2}}\right)-e^{\frac{1}{2} u} f_{1}^{1 / 2}\left(e^{u}\right)\right\}^{2} d u \times \mathcal{I}_{\phi}^{2}\left(f_{1}\right)\left(m(\bar{x})^{2}-\overline{x^{2}}\right)\right] .
\end{aligned}
$$

Since $e^{\frac{1}{2} u} f_{1}^{1 / 2}\left(e^{u}\right), e^{\frac{1}{2} u} f_{1}^{1 / 2}\left(e^{u}\right) \phi_{f_{1}}\left(e^{u}\right)$ and $\phi_{f_{1}}\left(e^{u}\right)$ are square integrable, then (A.13) is $o(1)$ as $h \longrightarrow 0$. 
With regard to $Q_{3}$, note that $Q_{3} \leq D\left(Q_{3}^{1}+Q_{3}^{2}\right)$ where

$$
\begin{aligned}
Q_{3}^{1} & :=\int_{\mathbb{R}^{m}}\left\{\frac{1}{4 \sigma^{2}}\left[\prod_{j=1}^{m} \frac{1}{\left(\sigma^{2}\right)^{1 / 4}} f_{1}^{1 / 2}\left(\frac{z_{j}}{\sigma}\right)\right]\left\{\sum_{j=1}^{m}\left[\psi_{f_{1}}\left(\frac{z_{j}}{\left(\sigma^{2}+v\right)^{1 / 2}}\right)-\psi_{f_{1}}\left(\frac{z_{j}}{\sigma}\right)\right] x_{j}^{2}\right\}\right\}^{2} d \mathbf{z} \\
& \leq D_{1} \int_{\mathbb{R}}\left\{\psi_{f_{1}}\left(e^{u-\ln \left(1+\frac{v}{\sigma^{2}}\right)^{1 / 2}}\right)-\psi_{f_{1}}\left(e^{u}\right)\right\}^{2} e^{u} f_{1}\left(e^{u}\right) d u=o(1) \text {, as } h \longrightarrow 0,
\end{aligned}
$$

and

$$
\begin{aligned}
Q_{3}^{2} & :=\int_{\mathbb{R}^{m}}\left\{\frac { 1 } { 4 \sigma ^ { 2 } } [ \prod _ { j = 1 } ^ { m } \frac { 1 } { ( \sigma ^ { 2 } ) ^ { 1 / 4 } } f _ { 1 } ^ { 1 / 2 } ( \frac { z _ { j } } { \sigma } ) ] \left\{\sum _ { j = 1 } ^ { m } \sum _ { \substack { l = 1 \\
l \neq j } } ^ { m } \left[\phi_{f_{1}}\left(\frac{z_{j}}{\left(\sigma^{2}+v\right)^{1 / 2}}\right) \phi_{f_{1}}\left(\frac{z_{l}}{\left(\sigma^{2}+v\right)^{1 / 2}}\right)\right.\right.\right. \\
& \left.\left.\left.-\phi_{f_{1}}\left(\frac{z_{j}}{\sigma}\right) \phi_{f_{1}}\left(\frac{z_{l}}{\sigma}\right)\right] x_{j} x_{l}\right\}\right\}^{2} d \mathbf{z} \\
& \leq D_{2} \int_{\mathbb{R}}\left\{\phi_{f_{1}}\left(e^{u-\ln \left(1+\frac{v}{\sigma^{2}}\right)^{1 / 2}}\right)-\phi_{f_{1}}\left(e^{u}\right)\right\}^{2} e^{u} f_{1}\left(e^{u}\right) d u=o(1), \text { as } h \longrightarrow 0 .
\end{aligned}
$$

Since $\psi_{f_{1}}\left(e^{u}\right)$ and $\phi_{f_{1}}\left(e^{u}\right)$ are square integrable. This completes the proof of Lemma A.1, and therefore, that of Proposition 2.1. 